\title{
Colored dark matter
}

\author{
Valerio De Luca, ${ }^{1}$ Andrea Mitridate, ${ }^{2}$ Michele Redi, ${ }^{3}$ Juri Smirnov, ${ }^{3}$ and Alessandro Strumia ${ }^{1,4}$ \\ ${ }^{1}$ Department of Physics "E. Fermi”, University of Pisa, Largo Bruno Pontecorvo 3, Pisa I-56127, Italy \\ ${ }^{2}$ Scuola Normale Superiore and INFN, Piazza dei Cavalieri 7, Pisa IT-56125, Italy \\ ${ }^{3}$ INFN and Department of Physics and Astronomy, University of Florence, \\ Via G. Sansone 1, Sesto Fiorentino I-50019, Italy \\ ${ }^{4}$ Theoretical Physics Department, CERN, 1211 Geneva 23, Switzerland
}

(Received 15 January 2018; published 14 June 2018)

\begin{abstract}
We explore the possibility that dark matter (DM) is the lightest hadron made of two stable color octet Dirac fermions $\mathcal{Q}$. The cosmological DM abundance is reproduced for $M_{\mathcal{Q}} \approx 12.5 \mathrm{TeV}$, compatibly with direct searches (the Rayleigh cross section, suppressed by $1 / M_{\mathcal{Q}}^{6}$, is close to present bounds), indirect searches (enhanced by $\mathcal{Q} \mathcal{Q}+\overline{\mathcal{Q}} \overline{\mathcal{Q}} \rightarrow \mathcal{Q} \overline{\mathcal{Q}}+\mathcal{Q} \overline{\mathcal{Q}}$ recombination), and with collider searches (where $\mathcal{Q}$ manifests as tracks, pair produced via QCD). Hybrid hadrons, made of $\mathcal{Q}$ and of standard model quarks and gluons, have large QCD cross sections, and do not reach underground detectors. Their cosmological abundance is $10^{5}$ times smaller than DM, such that their unusual signals seem compatible with bounds. Those in the Earth and stars sank to their centers; the Earth crust and meteorites later accumulate a secondary abundance, although their present abundance depends on nuclear and geological properties that we cannot compute from first principles.
\end{abstract}

DOI: 10.1103/PhysRevD.97.115024

\section{INTRODUCTION}

Many models of particle dark matter (DM) have been proposed; one common feature is that DM is a new neutral and uncolored particle. We challenge this view: can DM be instead colored or charged, and be dominantly present today in the form of neutral bound states kept together by ordinary electromagnetic or strong interactions analogously to hydrogen or neutrons? The answer is no for electric binding: two charged particles with mass $M \gg m_{e}$ form a negligible amount of neutral bound states, when their thermal relic abundance matches the DM cosmological abundance.

On the other hand, colored particles necessarily form hadronic bound states. We add to the standard model (SM) a new stable heavy colored particle $\mathcal{Q}$, for simplicity neutral. $\mathcal{Q}$ could be a heavy quark in the $3 \oplus \overline{3}$ representation of SU(3) $)_{c}$, or a "Dirac gluino" in the $8 \oplus 8$ representation, such that $\mathcal{Q}$ annihilates with $\overline{\mathcal{Q}}$, but not with itself. We dub this neutral quark as quorn. Perturbative annihilations and recombination between $\mathcal{Q}$ and $\overline{\mathcal{Q}}$ leave a thermal relic density of order $\Omega_{\mathcal{Q}} h^{2} \sim 0.1 M_{\mathcal{Q}} / 7 \mathrm{TeV}$.

Published by the American Physical Society under the terms of the Creative Commons Attribution 4.0 International license. Further distribution of this work must maintain attribution to the author(s) and the published article's title, journal citation, and DOI. Funded by SCOAP ${ }^{3}$.
After the QCD phase transition at temperature $T \lesssim$ $\Lambda_{\mathrm{QCD}} \approx 0.27 \mathrm{GeV}$ colored particles bind in hadrons. Subsequent annihilations among hadrons reduce their relic abundance, increasing the value of $M_{\mathcal{Q}}$ such that DM has the observed cosmological abundance, $\Omega_{\mathrm{DM}} h^{2} \sim$ 0.1 for $M_{\mathcal{Q}} \approx 10 \mathrm{TeV}$.

The quorn-onlyum hadrons made of $\mathcal{Q}$ only $(\mathcal{Q Q}$ if $\mathcal{Q} \sim 8$, and $\mathcal{Q} \mathcal{Q} \mathcal{Q}$ if $\mathcal{Q} \sim 3$ ) are acceptable DM candidates, as they have a small Bohr-like radius $a \sim 1 / \alpha_{3} M_{\mathcal{Q}}$. This scenario is believed to be excluded because it predicts other hybrid hadrons where $\mathcal{Q}$ binds with SM quarks $q$ or gluons $g$. Such hybrids, $\mathcal{Q} q q, \mathcal{Q} \mathcal{Q} q, Q \bar{q}$ (if $\mathcal{Q} \sim 3$ ) and $\mathcal{Q} g, \mathcal{Q} q \bar{q}^{\prime}$ (if $\mathcal{Q} \sim 8$ ), have size of order $1 / \Lambda_{\mathrm{QCD}}$ and thereby cross sections of order $\sigma_{\mathrm{QCD}} \sim 1 / \Lambda_{\mathrm{QCD}}^{2}$, can be charged, and are subject to strong bounds. Their cosmological abundance must be orders of magnitude smaller than the DM abundance $\Omega_{\mathrm{DM}} \approx 0.1$, while naively one might expect that cosmological evolution results in $\Omega_{\text {hybrid }} \gg \Omega_{\mathrm{DM}}$, given that quarks and gluons are much more abundant than quorns $\mathcal{Q}$.

We show that cosmological evolution gives $\Omega_{\text {hybrid }} \sim$ $10^{-4} \Omega_{\mathrm{DM}}$, such that this scenario is allowed. This is not surprising, taking into account that quorn onlyum has a binding energy $E_{B} \sim \alpha_{3}^{2} M_{\mathcal{Q}} \sim 200 \mathrm{GeV}$ much larger than hybrids, $E_{B} \sim \Lambda_{\mathrm{QCD}}$. Quorn onlyum thereby is the ground state, reached by the Universe if it has enough time to thermalize. This depends on two main factors. 
(i) Quorns are much rarer than quarks and gluons: $n_{\mathcal{Q}} \sim 10^{-14} n_{q, g}$ when the DM abundance is reproduced;

(ii) QCD interactions are much faster than the Hubble rate $H \sim T^{2} / M_{\mathrm{Pl}}$ : a loose bound state with a $\sigma_{\mathrm{QCD}}$ cross section recombines $N \sim n_{q, g} \sigma_{\mathrm{QCD}} / H \sim$ $M_{\mathrm{Pl}} / \Lambda_{\mathrm{QCD}} \sim 10^{19}$ times in a Hubble time at temperature $T \sim \Lambda_{\mathrm{QCD}}$.

Since $10^{19}$ is much bigger than $10^{14}$, chromodark synthesis cosmologically results in quorn onlyum plus traces of hybrids. This is analogous to big bang (BBN) nucleosynthesis, which leads to the formation of deeply bounded Helium plus traces of deuterium and tritium.

The paper is organized as follows. In Sec. II we define the model, and summarize the main features of its QCD interactions. In Sec. III we discuss how cosmology leads to dominant formation of $\mathcal{Q}$-onlyum hadrons. In Sec. IV we show that the abundance of hybrids is small enough to be compatible with bounds. In Sec. V we show that $\mathcal{Q}$-onlyum DM is compatible with bounds. A summary of our results is given in the conclusions in Sec. VI.

\section{THE MODEL}

We consider the following extension of the $\mathrm{SM}^{1}$ :

$$
\mathcal{L}=\mathcal{L}_{\mathrm{SM}}+\overline{\mathcal{Q}}\left(i \not D-M_{\mathcal{Q}}\right) \mathcal{Q}
$$

The only new ingredient is $\mathcal{Q}$ : a Dirac fermion with quantum numbers $(8,1)_{0}$ under $\mathrm{SU}(3)_{c} \otimes \mathrm{SU}(2)_{L} \otimes$ $\mathrm{U}(1)_{Y}$, i.e., a neutral color octet. The only free parameter is its mass $M_{\mathcal{Q}}$. Like in minimal dark matter models [3] $\mathcal{Q}$ is automatically stable, as no renormalizable interaction with SM particles allows its decay, which can first arise due to dimension-6 effective operators such as $\mathcal{Q} D D U$ and $\mathcal{Q} L D Q$ where $Q(L)$ is the SM quark (lepton) doublet, and $U(D)$ is the right-handed SM up-type (down-type) quark. The decay rate is cosmologically negligible if such operators are suppressed by the Planck scale.

After confinement $\mathcal{Q}$ forms bound states. For $M_{\mathcal{Q}} \gg$ $\Lambda_{\mathrm{QCD}} / \alpha_{3}$ states made by $\mathcal{Q}$ only are Coulombian. The $\mathcal{Q} \overline{\mathcal{Q}}$ bound states are unstable: $\mathcal{Q}$ and $\bar{Q}$ annihilate into gluons and quarks. No such annihilation arises in $\mathcal{Q} \mathcal{Q}$ bound states as we assumed that $\mathcal{Q}$ carries an unbroken $\mathrm{U}(1)$ dark baryon number that enforces the Dirac structure such that $\mathcal{Q Q}$ is stable. The DM candidate is the quorn-onlyum $\mathcal{Q} \mathcal{Q}$ ground state, neutral, colorless and

\footnotetext{
${ }^{1}$ Within the SM, QCD could give rise to dark matter as "strangelets" made of many $u d s$ quarks [1] or as "sexaquark" uuddss [2]. However there is neither experimental nor lattice evidence that such objects exist. We thereby extend the SM.
}

with spin $0 .^{2}$ As we see, if $\mathcal{Q Q}$ is a thermal relic, the observed cosmological DM abundance is reproduced for $M_{\mathcal{Q}} \sim 12.5 \mathrm{TeV}$. This mass is large enough that $\mathcal{Q}$ does not form QCD condensates. The $\mathcal{Q Q}$ potential in the color-singlet channel is $V(r)=-3 \alpha_{3} / r$, so the binding energy is $E_{B}=9 \alpha_{3}^{2} M_{\mathcal{Q}} / 4 n^{2} \approx 200 \mathrm{GeV} / n^{2}$, which is bigger than $\Lambda_{\mathrm{QCD}}$ up to $n \sim 20$. We adopt the value $\Lambda_{\mathrm{QCD}} \approx 0.27 \mathrm{GeV}$.

The quantum numbers of the hybrid hadrons, $\mathcal{Q} g$ and $\mathcal{Q} q \bar{q}^{\prime}$, are not exotic. We expect that the isospin singlet $\mathcal{Q} g$ is lighter than $\mathcal{Q} q \bar{q}^{\prime}$ (isospin $3 \oplus 1$ ) by an amount of order $\Lambda_{\mathrm{QCD}}$, which accounts for the relative motion of $q$ and $\bar{q}^{\prime}$, where $q, q^{\prime}=\{u, d\}$. A lattice computation is needed to safely establish who is lighter. Assuming that $\mathcal{Q} q \bar{q}^{\prime}$ is heavier, then its neutral component $\mathcal{Q} q \bar{q}$ decays to $\mathcal{Q} g$ with a lifetime of order $1 / \Lambda_{\mathrm{QCD}}$. The slightly heavier components $\mathcal{Q} u \bar{d}$ and $\mathcal{Q} d \bar{u}$ with electric charges \pm 1 have a lifetime of order $v^{4} / \Lambda_{\mathrm{QCD}}^{5}$.

The above DM model has possible extra motivations. The fermion $\mathcal{Q}$ appears as a Dirac gluino in some $N=2$ supersymmetric models [5], where sfermions can mediate its decay, if $R$-parity is broken. Alternatively, the heavy quarks $\mathcal{Q}$ could be identified with those introduced in Kim-ShifmanVainshtein-Zakharov axion models [6]. In such a case our U(1) symmetry gets related to the Peccei-Quinn symmetry. Corrections to the Higgs mass squared proportional to $M_{\mathcal{Q}}^{2}$ arise at three loops and are comparable to its measured value for $M_{\mathcal{Q}} \approx 10 \mathrm{TeV}$ [7].

\section{A. Confinement}

QCD confinement happens in cosmology through a smooth crossover. In Cornell parametrization [8] the QCD potential between two quarks in the $F$ fundamental representation at temperature $T$ in the singlet configuration is approximated as $V_{q \bar{q}}(r) \approx-\alpha_{F \text { eff }} / r+\sigma_{F} r$. In the perturbative limit one has $\alpha_{F \text { eff }}=C_{F} \alpha_{3}$ where $C_{F}=\left(N_{c}^{2}-1\right) /$ $2 N_{c}=4 / 3$ is the quadratic Casimir and $\alpha_{3}$ is renormalized around $1 / r$. At $r \sim 1 / \Lambda_{\mathrm{QCD}}$ lattice simulations find $\alpha_{F \text { eff }}=$ 0.4 and $\sigma_{F} \approx(0.45 \mathrm{GeV})^{2}$ [9]. The potential between two adjoints is similarly approximated by a Coulombian term plus a flux tube,

$$
V_{\mathcal{Q Q}}(r) \approx-\frac{\alpha_{\mathrm{eff}}}{r}+\sigma r
$$

\footnotetext{
${ }^{2}$ Other assignments of quantum numbers of $\mathcal{Q}$ are possible. A scalar would give similar physics. A fermionic $\mathcal{Q} \sim(3 \oplus \overline{3}, 1)_{0}$ under $\mathrm{SU}(3)_{c} \otimes \mathrm{SU}(2)_{L} \otimes \mathrm{U}(1)_{Y}$ would give the $\mathcal{Q} \mathcal{Q} \mathcal{Q}$ baryon as a viable DM candidate. As the gauge quantum numbers of a neutral color triplet are exotic, the $\mathcal{Q} \mathcal{Q} q, \mathcal{Q} q q$ and $\mathcal{Q} \bar{q}$ hadrons containing light quarks would have fractional charges. Fractionally charged hadrons are subject to stronger experimental bounds [4]. A $\mathcal{Q} \sim(3,2,1 / 6)=\left(\mathcal{Q}_{u}, \mathcal{Q}_{d}\right)$, with the same quantum numbers of SM left-handed quarks $Q$, would give as the lightest state the neutral DM candidate $\mathcal{Q}_{u} \mathcal{Q}_{d} \mathcal{Q}_{d}$. This is excluded by direct detection mediated at tree level by a $Z$, being a weak doublet with hypercharge $Y \neq 0$. Allowing for an additional confining group, a $\mathcal{Q} \sim 8$ can be built out of $\mathcal{Q} \sim 3$ obtaining double composite dark matter.
} 
Perturbation theory implies $V_{\mathcal{Q Q}} / C_{A} \approx V_{q \bar{q}} / C_{F}$ [10] where $C_{A}=N_{c}=3$. Thereby $\alpha_{\text {eff }} \approx 3 \alpha_{3}$ and $\sigma(0) \approx 9 \sigma_{F}(0) / 4 \approx$ $(0.67 \mathrm{GeV})^{2}$, as verified on the lattice [11]. At finite temperature the Coulombian force gets screened by the Debye mass and the string appears only below the critical temperature $T_{c} \approx 170 \mathrm{MeV}$ as $\sigma(T) \approx \sigma(0) \sqrt{1-T^{2} / T_{c}^{2}}$ [9].

\section{B. Eigenvalues in a linear plus Coulombian potential}

We need the binding energies of a nonrelativistic $\mathcal{Q Q}$ hadron. We thereby consider the Hamiltonian $H=$ $\vec{p}^{2} / 2 \mu+V(r)$ in three dimensions that describes its motion around the center of mass, with reduced mass $2 \mu \simeq M_{\mathcal{Q}}$. The potential is given by Eq. (2). As usual, wave functions are decomposed in partial waves as $\psi(r, \theta, \phi)=$ $\sum_{\tilde{n}, \ell, m} R_{\tilde{n} \ell}(r) Y_{\ell m}(\theta, \phi)$ where $\tilde{n}$ is the principal quantum number. For each $\ell=0,1,2, \ldots$ we define as $\tilde{n}=1$ the state with lowest energy, so that $\tilde{n}=1,2,3, \ldots$. The radial wave function $R_{\tilde{n} e}(r)$ has $\tilde{n}-1$ nodes. Unlike in the hydrogen atom there are no free states: angular momentum $\ell$ is not restricted to $\ell<\tilde{n}$. In order to match with the Coloumbian limit in its usual notation we define $n \equiv \tilde{n}+\ell$ such that, at given $\ell$, only $n \geq \ell+1$ is allowed.

The reduced wave function $u_{\tilde{n} \ell}(r)=r R_{\tilde{n} \ell}(r)$ obeys the Schroedinger equation in one dimension in the effective potential $V_{\text {eff }}=V+\ell(\ell+1) \hbar^{2} / 2 \mu r^{2}$. Rescaling arguments imply that energy eigenvalues have the form

$$
E_{\tilde{n} \ell}=\alpha_{\mathrm{eff}}^{2} \mu \times f(\varepsilon, \tilde{n}, \ell),
$$

where $\varepsilon \equiv \frac{\sigma}{4 \alpha_{\mathrm{eff}}^{3} \mu^{2}}=10^{-8} \frac{\sigma}{\mathrm{GeV}^{2}}\left(\frac{10 \mathrm{TeV}}{M_{\mathcal{Q}}}\right)^{2}\left(\frac{1}{\alpha_{\mathrm{eff}}}\right)^{3}$.

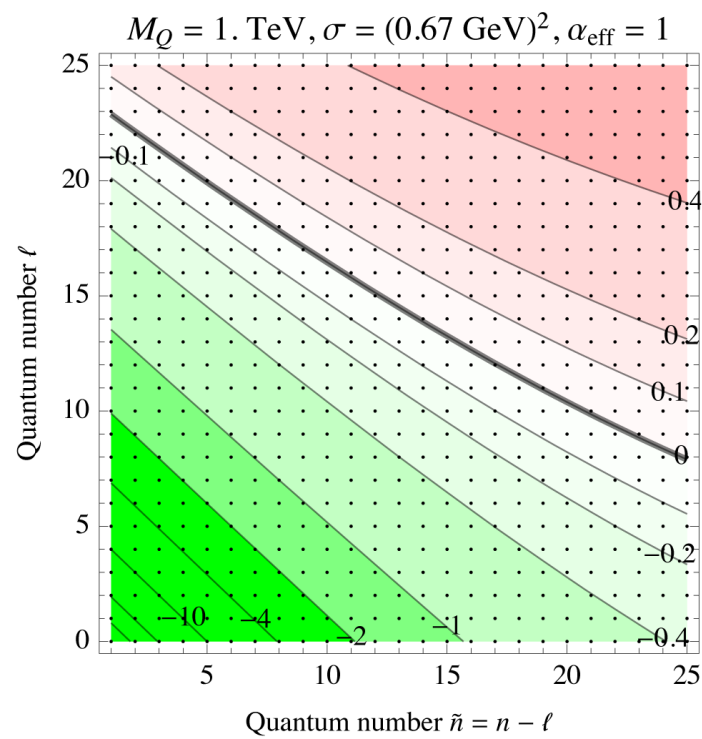

From [12] we extract the approximation valid at leading order in $\varepsilon \ll 1$,

$$
E_{\tilde{n} \ell}=\frac{\alpha_{\mathrm{eff}}^{2} \mu}{2}\left[-\frac{1}{n^{2}}+8 \varepsilon n(\ell+1.37)+\cdots\right] .
$$

The first term is Coulombian. The second term accounts for the linear potential, and becomes relevant at large $n, \ell$. In particular, assuming $\ell \simeq n \gg 1$, Coulombian states with negative binding energy exist up to $\ell<\alpha_{\text {eff }}^{3 / 4} M_{\mathcal{Q}}^{1 / 2} /(8 \sigma)^{1 / 4}$. The ground state has binding energy $E_{B}=-E_{10} \sim$ $200 \mathrm{GeV}$ for $M_{\mathcal{Q}} \sim 10 \mathrm{TeV}$.

In the opposite limit where the linear force dominates and the Coulomb-like force can be neglected, all energy levels are positive and states with higher $\ell$ have higher energy [12],

$$
E_{\tilde{n} \ell} \approx \frac{3 \sigma^{2 / 3}}{(2 \mu)^{1 / 3}}\left(0.897 \tilde{n}+\frac{\ell}{2}-0.209\right)^{2 / 3},
$$

such that thermalization lowers $\ell$. The dependence on $\sigma, \mu$ and the ground state energy can also be computed variationally, assuming a trial wave function $\psi(r)=e^{-r / r_{c}} / r_{c}^{3 / 2}$, such that the typical size is $r_{c} \sim(\mu \sigma)^{-1 / 3}$. Figure 1 shows the binding energies for relevant values of the parameters.

We next discuss a bound state $B_{\mathcal{Q}}$ made of a heavy $\mathcal{Q}$ and a gluon. It cannot be described by nonrelativistic quantum mechanics. Nevertheless, its binding energy can roughly be obtained by Eq. (5) taking a small reduced mass $\mu \sim \sqrt{\sigma}$. One then expects that such states are in their ground states at $T \lesssim \Lambda_{\mathrm{QCD}}$, and that their mass is $M_{B_{\mathcal{Q}}}=M_{\mathcal{Q}}+\mathcal{O}\left(\Lambda_{\mathrm{QCD}}\right)$.

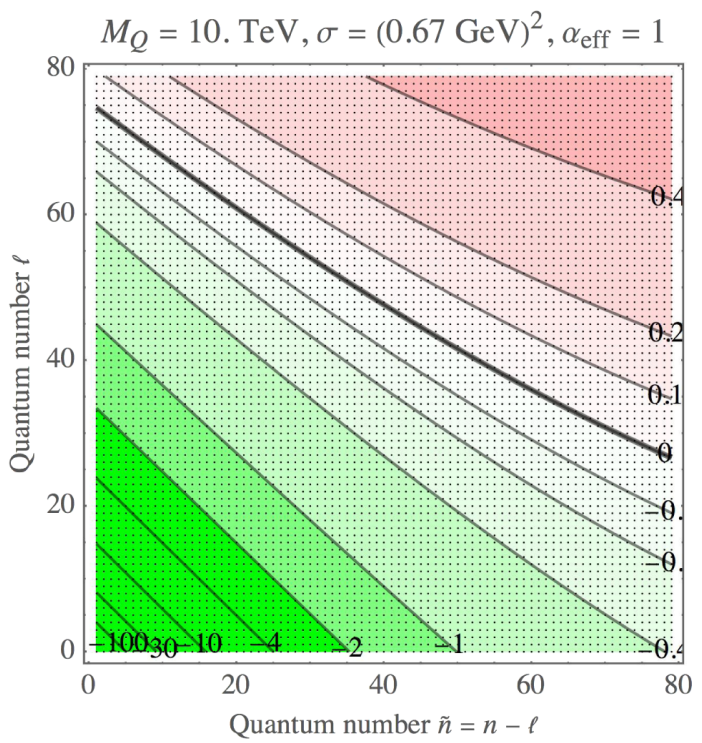

FIG. 1. Binding energies $E_{\tilde{n} \ell}$ in $\mathrm{GeV}$ for a $\mathcal{Q} \mathcal{Q}$ in the singlet configuration. States with $E_{\tilde{n} \ell}<-0.2 \mathrm{GeV}$ (in green) are well approximated by the Coulombian limit. Increasing $M_{\mathcal{Q}}$ leads to a larger number of Coulombian states and to a deeper ground state. $\mathcal{Q} \mathcal{Q}$ states are cosmologically mostly produced in the region with larger $\ell$ of the band $E \sim \Lambda_{\mathrm{QCD}}$. 


\section{Decay rates of excited bound states}

Energy losses due to quantum decay of a $\mathcal{Q Q}$ state with $n, \ell \gg 1$ into deeper states can be approximated with classical Larmor radiation. This holds in dipole approximation, where a state can only decay to $\ell^{\prime}=\ell \pm 1$.

To see this, we consider a hydrogenlike system with $V=-\alpha / r$ and reduced mass $\mu$. Assuming a circular orbit as in [13] one gets the emitted power

$$
W_{\text {Larmor }}^{\text {circ }}=\frac{2 \alpha a^{2}}{3}=\frac{2 \mu^{2} \alpha^{7}}{3 n^{8}}
$$

having inserted the acceleration $a=\alpha / \mu r^{2}$ and converted the orbital radius into $n^{2}$ times the Bohr radius as $r=$ $r_{n}=n^{2} / \alpha \mu$. Similarly, the binding energy is $E=-\alpha / 2 r=$ $-\alpha^{2} \mu / 2 n^{2}$.

At quantum level, a circular orbit corresponds to a state with maximal $\ell=\ell_{\text {circ }}=n$. In dipole approximation such a state decays only to $n^{\prime}=\ell^{\prime}=n-1$, emitting a soft photon with energy $\Delta E_{\text {Larmor }}=\left|E_{n}-E_{n-1}\right| \simeq \alpha^{2} \mu / n^{3}$, such that the decay rate is

$$
\Gamma_{\text {Larmor }}^{\text {circ }}=\frac{W_{\text {Larmor }}^{\text {circ }}}{\left|\Delta E_{\text {Larmor }}\right|}=\frac{2}{3}\left(\frac{\alpha}{n}\right)^{5} \mu .
$$

This matches the quantum decay rate.

Let us now consider a generic state. Classically, a generic elliptic orbit is parametrized by its energy $E$ and by its angular momentum $\ell \leq \ell_{\text {circ }}$, where $\ell_{\text {circ }}=\sqrt{\alpha^{2} \mu / 2 E}$ is the value corresponding to a circular orbit. The Larmor radiation power, averaged over the orbit, is

$$
\left\langle W_{\text {Larmor }}\right\rangle=W_{\text {Larmor }}^{\text {circ }} \frac{3-\left(\ell / \ell_{\text {circ }}\right)^{2}}{2\left(\ell / \ell_{\text {circ }}\right)^{5}} .
$$

Due to the larger acceleration at the point of minimal distance, the radiated energy for $\ell \ll \ell_{\text {circ }}$ is much larger than in the circular case: this is why $e \bar{e}$ colliders are built circular.

This classical result for noncircular orbits agrees with the quantum results for $n, \ell \gg 1$, summarized in Appendix A for the hydrogen atom, which can be approximated as

$$
\Gamma_{n \ell} \simeq \frac{2 \alpha^{5} \mu}{3 n^{3} \ell^{2}}, \quad W_{n \ell} \simeq \frac{2 \alpha^{7} \mu^{2}}{3 n^{8}} \frac{3-(\ell / n)^{2}}{2(\ell / n)^{5}} .
$$

In the quantum computation the enhancement at small $\ell<n$ appears after summing over the available final states with small $n^{\prime} \geq \ell-1$ which allows for energy jumps $\left|E_{n}-E_{n^{\prime}}\right|$ larger than in the circular case.

In the opposite limit where the linear part of the potential dominates over the Coulombian part, energy losses of highly excited states are again well approximated by classical Larmor radiation, which does not depend on the shape of the orbit, given that the force does not depend on the radius: $W_{\text {Larmor }}=8 \alpha_{\text {eff }} \sigma^{2} / 3 M_{\mathcal{Q}}^{2}$ is negligibly small. This is confirmed by numerical quantum computations.

\section{Cross section for formation of a loose $\mathcal{Q} \mathcal{Q}$ bound state}

We here estimate the cross section $\sigma_{\text {tot }}\left(B_{\mathcal{Q}}+B_{\mathcal{Q}} \rightarrow\right.$ $\left.B_{\mathcal{Q Q}}+X\right)$ for formation of a loose bound state containing two heavy quarks $\mathcal{Q}$, starting from two bound states $B_{\mathcal{Q}}$ containing one $\mathcal{Q}$.

Assuming that $B_{\mathcal{Q}}=\mathcal{Q} g$ can be approximated as a $\mathcal{Q}$ and a gluon kept together by a flux tube with length $\ell \sim 1 / \Lambda_{\mathrm{QCD}}$, the following geometrical picture emerges. The cross section is $\sigma_{\text {tot }} \approx \pi \ell^{2} \wp$ at energies $E \sim M_{\mathcal{Q}} v^{2} \lesssim \Lambda_{\mathrm{QCD}}$ such that there is not enough energy for breaking the QCD flux tubes, and the recombination probability of two flux tubes is $\wp \sim 1$, like in string models. Independently from the above geometric picture, the size of the bound state is of order $1 / \Lambda_{\mathrm{QCD}}$, and thereby one expects a cross section $\sigma_{\mathrm{QCD}}=c / \Lambda_{\mathrm{QCD}}^{2}$, with $c \approx \pi$ in the geometric picture. In the following we consider $c=\{1, \pi, 4 \pi\}$. For example the measured $p p$ cross section corresponds to $c \approx 10$.

While this expectation is solid at energies of order $\Lambda_{\mathrm{QCD}}$, at lower temperatures the cross section might be drastically suppressed if the residual van der Waals-like force has a repulsive component, which prevents the particles coming close enough. We ignore this possibility, which would result into a higher abundance of hybrid relics.

More in general, processes that only require a small energy exchange $E$ can have large cross sections of order $1 / E^{2}{ }^{3}$.

\section{E. Cross section for formation of an unbreakable $\mathcal{Q} \mathcal{Q}$ bound state}

We can finally compute the quantity of interest for us: the thermally averaged cross section $\sigma_{\text {fall }}(T)$ for collisions between two $\mathcal{Q} g$ states which produce an unbreakable $\mathcal{Q} \mathcal{Q}$ hadron. This happens when the loose bound state discussed in the previous section radiates more energy than $\sim T$ in the time $\Delta t$ before the next collision, such that it becomes unbreakable and later falls down to its deep ground state.

In view of the previous discussion, we proceed as follows. A large total cross section $\sigma_{\mathrm{QCD}} \sim \pi / \Lambda_{\mathrm{QCD}}^{2}$ needs a large impact parameter $b \sim 1 / \Lambda_{\mathrm{QCD}}$, and thereby the $\mathcal{Q Q}$ state is produced with large angular momentum $\ell \sim M_{\mathcal{Q}} v b$.

The issue is whether a bound state with large $\ell$ gets broken or radiates enough energy becoming unbreakable [13]. As discussed in Sec. II C, Abelian energy losses are well approximated by classical Larmor radiation, and it is crucial to take into account that noncircular orbits radiate

\footnotetext{
${ }^{3}$ The authors of [14] propose a quantum mechanical model where processes analogous to $\sigma\left(B_{\mathcal{Q}}+B_{\mathcal{Q}} \rightarrow B_{\mathcal{Q Q}}+X\right)$ are computed in terms of cross sections suppressed by $1 / M_{\mathcal{Q}}$. This large suppression seems to derive from their arbitrary assumption that the cross section should be dominated by an $s$-channel resonance.
} 
much more than circular orbits. The $\mathcal{Q} \mathcal{Q}$ potential is given by Eq. (2), with a large $\alpha_{\text {eff }} \approx 3 \alpha_{3}(\bar{\mu})$ renormalized at $\bar{\mu} \sim 1 / r \sim \Lambda_{\mathrm{QCD}}{ }^{4}$

The cross section for falling into an unbreakable $\mathcal{Q Q}$ bound state is computed as follows. We simulate classical collisions, averaging over the velocity distribution at temperature $T$ and over the impact parameter $b$. We numerically solve the classical equation of motion for the $\mathcal{Q Q}$ system, starting from an initial relative distance $b$ and an orthogonal relative velocity $v$. From the solution $\vec{x}(t)$ we compute the radiated energy $\Delta E$ by integrating the radiated power $W_{\text {Larmor }} \sim 2 \alpha_{\text {eff }} \ddot{\vec{x}}^{2} / 3$ for a time $\Delta t$. We impose $\Delta E \gtrsim T$ where $\Delta t$ is the average time between two collisions at temperature $T$. We estimate it as $\Delta t \sim$ $1 / n_{\pi} v_{\pi} \sigma_{\mathrm{QCD}}$ where $n_{\pi}$ is the pion number density and $\sigma_{\mathrm{QCD}}=c / \Lambda_{\mathrm{QCD}}^{2}$ such that $\Delta t \simeq \Lambda_{\mathrm{QCD}}^{2} / T^{3}$ at $T \gg m_{\pi}$, while the pion density is Boltzmann suppressed at lower $T$.

The resulting $\sigma_{\text {fall }}(T)$ is plotted in Fig. 2, computed varying the uncertain QCD parameters as $\alpha_{\text {eff }}$, $c=\{1, \pi, 4 \pi\}$. We see that even for $\alpha_{\text {eff }} \sim 1$ the fall cross section $\sigma_{\text {fall }}(T)$ equals to the total cross section $\sigma_{\mathrm{QCD}}$ at temperatures below $(0.1-0.3) \Lambda_{\mathrm{QCD}}$, and it is mildly smaller at $T \sim \Lambda_{\mathrm{QCD}}$. If instead $\alpha_{\text {eff }} \sim 4 \pi$ one would have $\sigma_{\text {fall }}=\sigma_{\mathrm{QCD}}$ even at $T \sim \Lambda_{\mathrm{QCD}}$. The value $\alpha_{\mathrm{eff}} \sim 4 \pi$ can account for nonperturbative QCD effects: it is not unreasonable to think that the bound state can quickly radiate the maximal binding energy $E_{B} \sim 200 \mathrm{GeV}$ by emitting in one shot a hundred gluons with energy $E \sim 2 \mathrm{GeV}$ each.

A rough analytical estimate for $\sigma_{\text {fall }}(T)$ can be obtained as follows. As discussed above, states that radiate fast enough arise only in the Coulombian part of the potential. In view of Eq. (8), their energy loss rate is $W_{\text {Larmor }} \sim$ $\alpha_{\text {eff }}^{7} M_{\mathcal{Q}}^{2} / \ell^{8}$, which can be big enough only for relatively small $\ell \sim M_{\mathcal{Q}} b v$. Imposing $\Delta E \gtrsim T$ for $v \sim \sqrt{T / M_{\mathcal{Q}}}$ gives

$$
\sigma_{\text {fall }} \sim \frac{c}{\Lambda_{\mathrm{QCD}}^{2}} \min (1,0.3 A) \quad A=\frac{\alpha_{\mathrm{eff}}^{7 / 4} \Lambda_{\mathrm{QCD}}^{5 / 2}}{M_{\mathcal{Q}}^{1 / 2} T^{2}}
$$

\footnotetext{
${ }^{4}$ We do not know how to generalize Abelian Larmor radiation to gluon emission. While emission of one soft photon negligibly affects the state of the system, the situation is different for gluon emission: gluons are colored, so that emitting one gluon changes the potential: a singlet state becomes an octet. The only soft particles that can be radiated are $g g$ color singlets. Using perturbative QCD techniques [15] we computed the singlet-tosinglet transition rate through $g g$ emission (and subsequent hadronization) between Coulombian bound states, finding values not much below our naive Larmor estimate. However, we cannot apply such techniques to the states with large $n, \ell$ relevant for us, affected by confinement. Possibly, the Abelian Larmor result correctly approximates the rate for emitting soft gluons that remain within the bound state, such that the $\mathcal{Q} \mathcal{Q}$ singlet becomes a $(\mathcal{Q} g) \mathcal{Q}$ singlet. If this picture is correct, $\mathcal{Q} \mathcal{Q}$ would fall to a deep bound state by losing energy to a cloud of gluons that surrounds the bound state.
}

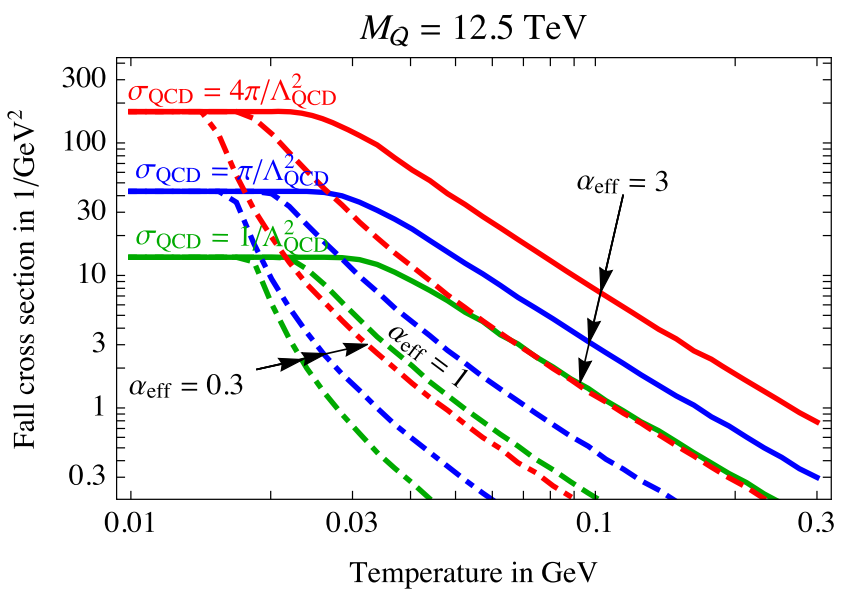

FIG. 2. Thermally averaged cross section for falling in an unbreakable bound state as computed numerically for $M_{\mathcal{Q}}=$ $12.5 \mathrm{TeV}$ and for different values of $\alpha_{\text {eff }}=0.3$ (dot-dashed) 1 (dashed), 3 (continuous) and for different values of the total $\mathrm{QCD}$ cross section, $\sigma_{\mathrm{QCD}}=c / \Lambda_{\mathrm{QCD}}^{2}, c=1$ (green), $\pi$ (blue), $4 \pi$ (red). Equation (10) approximates this numerical result.

where the order 1 numerical value was added by roughly fitting to Fig. 2, for the values of the total QCD cross section there assumed. The fall cross section is only suppressed by a small power of $M_{\mathcal{Q}}$, explaining why we find a large $\sigma_{\text {fall }} \sim \sigma_{\text {tot }}$ for $M_{\mathcal{Q}} \sim 12.5 \mathrm{TeV}$. In the analytic estimate we neglected the fact that $m_{\pi} \sim \Lambda_{\mathrm{QCD}}$ : this is taken into account by the relatively large $a d$ hoc numerical factor added to Eq. (10) such that it provides a better agreement with the numerical result in Fig. 2 for $M_{\mathcal{Q}} \sim 12.5 \mathrm{TeV}$.

\section{COSMOLOGICAL RELIC DENSITIES}

We can now compute how strong QCD interactions lead to an abundance of the $\mathcal{Q}$-onlyum DM candidate $\mathcal{Q} \mathcal{Q}$ much larger than the severely constrained hybrid bound states $\mathcal{Q} g$. We describe what happens during the cosmological evolution, from the usual decoupling of free $\mathcal{Q}$ at $T \sim$ $M_{\mathcal{Q}} / 25$ (Sec. III A), to recoupling (Sec. III B) at $T \gtrsim \Lambda_{\mathrm{QCD}}$, to $T \sim \Lambda_{\mathrm{QCD}}$ (Sec. III C), to redecoupling at $T \lesssim \Lambda_{\mathrm{QCD}}$ (Sec. III D), to nucleosynthesis at $T \sim 0.1 \mathrm{MeV}$ (Sec. III E).

\section{A. $\mathcal{Q}$ decoupling at $T \sim M_{\mathcal{Q}} / 25$}

As usual, at $T \gtrsim M_{\mathcal{Q}}$ the free $\mathcal{Q}$ annihilate into SM particles much faster than the Hubble rate, remaining in thermal equilibrium until they decouple at $T=T_{\mathrm{dec}} \approx$ $M_{\mathcal{Q}} / 25$, leaving the usual relic abundance, determined by their annihilation cross section in this decoupling phase. The nonrelativistic $s$-wave cross section reads

$$
\sigma_{\mathrm{ann}} v_{\mathrm{rel}}=\frac{\sigma_{\mathcal{Q} \overline{\mathcal{Q}}} v_{\mathrm{rel}}}{2}=\frac{63}{64}\left(\frac{1}{14} S_{3}+\frac{10}{14} S_{3 / 2}+\frac{3}{14} S_{-1}\right) \frac{\pi \alpha_{3}^{2}}{M_{\mathcal{Q}}^{2}},
$$


where the strong coupling is renormalized around $M_{\mathcal{Q}}$, while it is renormalized around $\alpha_{3} M_{\mathcal{Q}}$ in the Sommerfeld factors $S_{n}$ corresponding to the various color channels,

$$
S_{n}=\frac{2 \pi n \alpha_{3} / v_{\mathrm{rel}}}{1-e^{-2 \pi n \alpha_{3} / v_{\mathrm{rel}}}} .
$$

We define $Y_{\mathcal{Q}} \equiv\left(n_{\mathcal{Q}}+n_{\overline{\mathcal{Q}}}\right) / s$, where $s$ is the entropy density, and assume no dark baryon asymmetry, $n_{\mathcal{Q}}=n_{\overline{\mathcal{Q}}}$.

Bound-state formation gives an order 1 correction to the relic abundance, as discussed in Ref. [16], which considered Majorana gluinos. The bound states made by our Dirac gluinos can be divided into stable $\mathcal{Q} \mathcal{Q}$ or $\overline{\mathcal{Q}} \mathcal{Q}$ states that carry two units of dark baryon number, and unstable $\mathcal{Q} \overline{\mathcal{Q}}$ states, where $\mathcal{Q}$ and $\overline{\mathcal{Q}}$ annihilate. The latter come into spin- 0 and spin- 1 combinations, while the stable states have only the spin allowed by Fermi statistics: in particular, the singlet ground state has spin 0 . Among the unstable bound states the most relevant for the relic abundance at $T \gg$ $\Lambda_{\mathrm{QCD}}$ are the ones that decay faster and have larger binding energy. These are listed in Table I. The corresponding effective rates are plotted in Fig. 3. We only estimated the annihilation widths of those states that exist only as $\mathcal{Q} \overline{\mathcal{Q}}$; they are suppressed by $\mathcal{O}\left(\alpha_{3}^{2}\right)$ making these states negligible (the formation cross section does not depend on spin) unless numerical factors compensate for the suppression.

These rates determine a network of Boltzmann equations for the abundance of free $\mathcal{Q}$ and for the abundances $Y_{I}=$ $n_{I} / s$ of the various bound states $I$ as a function of $z=M_{\mathcal{Q}} / T$. In the notations of [16] such equations are

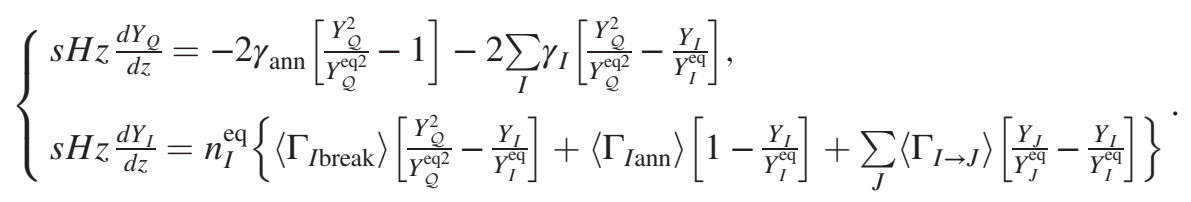

Here $\gamma_{I}$ is the thermal-equilibrium space-time density of formations of bound state $I$, related to the thermal average $\left\langle\Gamma_{\text {Ibreak }}\right\rangle$ of the breaking rate $\Gamma_{\text {Ibreak }}$ as described in [16]. Furthermore $\Gamma_{I \text { ann }}$ is the decay rate of bound state $I$ due to annihilations between its $\mathcal{Q}$ and $\overline{\mathcal{Q}}$ constituents: it vanishes for the $\mathcal{Q} \mathcal{Q}$ and $\overline{\mathcal{Q}} \overline{\mathcal{Q}}$ states. Finally, $\Gamma_{I \rightarrow J}=-\Gamma_{J \rightarrow I}$ is the decay rate from state $I$ to state $J$. Taking into account that the annihilation and decay rates are much larger than the Hubble rate, Ref. [16] used thermal-equilibrium conditions to substitute the network of Boltzmann equations with a single equation for the total DM density, in terms of an effective annihilation rate $\gamma_{\text {ann }}^{\text {eff }}$. This strategy needs to be extended including the $\mathcal{Q} \mathcal{Q}$ and $\overline{\mathcal{Q}} \overline{\mathcal{Q}}$ states. Their annihilation rates $\Gamma_{\text {ann }}$ vanish, so we can now only reduce the network of Boltzmann equations to two equations: one for $Y_{\mathcal{Q}}$ (density of free $\mathcal{Q}$ ) and one for $Y_{\mathcal{Q Q}}=\sum_{I \in \mathcal{Q} \mathcal{Q}} Y_{I}$ (total density of stable bound states, which satisfies $Y_{\mathcal{Q Q}} / Y_{\mathcal{Q Q}}^{\mathrm{eq}}=$ $Y_{I} / Y_{I}^{\mathrm{eq}}$ for all stable states $I$ ). The equations are

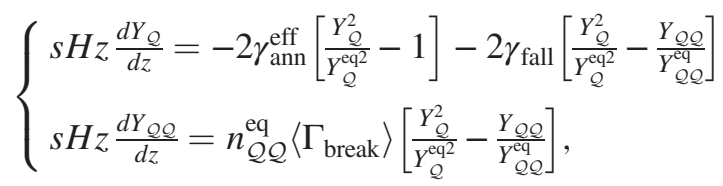

where $\gamma_{\text {ann }}^{\text {eff }}$ includes the effects of $\mathcal{Q} \overline{\mathcal{Q}}$ bound states and is given by the same expression as in [16]. The total fall rate that accounts for the cumulative effect of all $\mathcal{Q} \mathcal{Q}$ and $\overline{\mathcal{Q}} \overline{\mathcal{Q}}$ bound states is given by the sum of the formation rates of all such states, $\gamma_{\text {fall }}=\sum_{I \in \mathcal{Q} \mathcal{Q}} \gamma_{I}$, which equals $n_{\mathcal{Q} \mathcal{Q}}^{\mathrm{eq}}\left\langle\Gamma_{\text {break }}\right\rangle \equiv$ $\sum_{I \in \mathcal{Q} \mathcal{Q}}\left\langle\Gamma_{\text {Ibreak }}\right\rangle n_{I}^{\text {eq }}$. Notice that $Y_{\mathcal{Q}}+2 Y_{\mathcal{Q Q}}$ remains constant when a $\mathcal{Q Q}$ bound state is formed.

We now derive an approximated analytic solution by computing the deviation from equilibrium of the stable bound states. First, we appreciate that at temperatures at which the quorn annihilation goes out of equilibrium the second of the above equations is still in equilibrium and thus the effect of stable bound states can be ignored in the solution for the first equation. The asymptotic solution in this phase is

$$
\left\{\begin{array}{l}
Y_{\mathcal{Q}}(z) \approx\left[Y_{\mathcal{Q}}\left(z_{\mathrm{dec}}\right)^{-1}+\lambda \int_{z_{\mathrm{dec}}}^{z} \frac{\left\langle\sigma_{\text {ann }}^{\text {eff }} v_{\mathrm{rel}}\right\rangle}{z^{2}} d z^{\prime}\right]^{-1} \\
Y_{\mathcal{Q Q}}(z) \approx Y_{\mathcal{Q} \mathcal{Q}}^{0}(z)+\frac{1}{\lambda} Y_{\mathcal{Q}}^{1}(z)=Y_{\mathcal{Q}}(z)^{2} \frac{Y_{\mathcal{Q}}^{\mathrm{eq}}}{Y_{\mathcal{Q}}^{\mathrm{eq}}}+\frac{1}{\lambda} Y_{\mathcal{Q Q}}^{1}(z),
\end{array}\right.
$$

where $z_{\mathrm{dec}} \approx 25$ and $1 / \lambda=H /\left.s\right|_{T=M_{\mathcal{Q}}}$. Expanding in small $1 / \lambda$ one finds $Y_{\mathcal{Q Q}}^{1}(z)$ and determines the temperature at which $Y_{\mathcal{Q Q}}^{0}(z) \approx Y_{\mathcal{Q Q}}^{1}(z) / \lambda$, finding

$$
1 \approx \frac{\left\langle\Gamma_{\text {break }}\right\rangle M_{\mathcal{Q}}}{E_{B} H(T) z} \approx \frac{\left\langle\Gamma_{\text {break }}\right\rangle}{H\left(T \approx E_{B}\right)} .
$$

This gives the asymptotic solution for $\Lambda_{\mathrm{QCD}} \ll T \ll M_{\mathcal{Q}}$, 
TABLE I. Properties of lowest lying Coulombian bound states made of $\mathcal{Q} \overline{\mathcal{Q}}$ (upper) and $\mathcal{Q} \mathcal{Q}$ (lower). The subscript $S$ or $A$ denotes if the state is obtained as a symmetric or antisymmetric combination in color space. Slower rates have only been estimated.

\begin{tabular}{|c|c|c|c|c|c|c|c|c|}
\hline Made of & Color & $S$ & $n$ & $\ell$ & $E_{B} / M_{\mathcal{Q}}$ & $\Gamma_{\mathrm{ann}} / M_{\mathcal{Q}}$ & $\Gamma_{\mathrm{dec}} / M_{\mathcal{Q}}$ & Annihilation \\
\hline $\mathcal{Q \mathcal { Q }}$ & $1_{S}$ & 0 & 1 & 0 & $9 \alpha_{3}^{2} / 4$ & $243 \alpha_{3}^{5} / 2$ & 0 & $g g$ \\
\hline $\mathcal{Q} \overline{\mathcal{Q}}$ & $1_{S}$ & 1 & 1 & 0 & $9 \alpha_{3}^{2} / 4$ & $\sim \alpha_{3}^{7}$ & $\sim \alpha_{3}^{6}$ & gggg \\
\hline $\mathcal{Q} \overline{\mathcal{Q}}$ & $8_{A}$ & 1 & 1 & 0 & $9 \alpha_{3}^{2} / 16$ & $243 \alpha_{3}^{5} / 32$ & $\sim \alpha_{3}^{6}$ & $(q \bar{q})\left(H H^{*}\right)$ \\
\hline $\mathcal{Q \mathcal { Q }}$ & $8_{A}$ & 0 & 1 & 0 & $9 \alpha_{3}^{2} / 16$ & $\sim \alpha_{3}^{6}$ & $\sim \alpha_{3}^{6}$ & ggg \\
\hline $\mathcal{Q \mathcal { Q }}$ & $8_{S}$ & 0 & 1 & 0 & $9 \alpha_{3}^{2} / 16$ & $243 \alpha_{3}^{5} / 64$ & $\sim \alpha_{3}^{6}$ & $g g$ \\
\hline $\mathcal{Q \mathcal { Q }}$ & $8_{S}$ & 1 & 1 & 0 & $9 \alpha_{3}^{2} / 16$ & $\sim \alpha_{3}^{7}$ & $\sim \alpha_{3}^{6}$ & gggg \\
\hline $\mathcal{Q \mathcal { Q }}$ & $1_{S}$ & 0 & 2 & 0 & $9 \alpha_{3}^{2} / 16$ & $243 \alpha_{3}^{5} / 16$ & $\sim \alpha_{3}^{6}$ & $g g$ \\
\hline $\mathcal{Q} \overline{\mathcal{Q}}$ & $1_{S}$ & 1 & 2 & 0 & $9 \alpha_{3}^{2} / 16$ & $\sim \alpha_{3}^{7}$ & $\sim \alpha_{3}^{6}$ & gggg \\
\hline $\mathcal{Q \mathcal { Q }}$ & $8_{A}$ & 1 & 2 & 0 & $9 \alpha_{3}^{2} / 64$ & $243 \alpha_{3}^{5} / 256$ & $\sim \alpha_{3}^{6}$ & $(q \bar{q})\left(H H^{*}\right)$ \\
\hline $\mathcal{Q} \overline{\mathcal{Q}}$ & $8_{A}$ & 0 & 2 & 0 & $9 \alpha_{3}^{2} / 64$ & $\sim \alpha_{3}^{6}$ & $\sim \alpha_{3}^{6}$ & ggg \\
\hline $\mathcal{Q \mathcal { Q }}$ & $8_{S}$ & 0 & 2 & 0 & $9 \alpha_{3}^{2} / 64$ & $243 \alpha_{3}^{5} / 512$ & $\sim \alpha_{3}^{6}$ & $g g$ \\
\hline $\mathcal{Q} \overline{\mathcal{Q}}$ & $8_{S}$ & 1 & 2 & 0 & $9 \alpha_{3}^{2} / 64$ & $\sim \alpha_{3}^{7}$ & $\sim \alpha_{3}^{6}$ & gggg \\
\hline $\mathcal{Q} \overline{\mathcal{Q}}$ & $1_{S}$ & 0 & 2 & 1 & $9 \alpha_{3}^{2} / 16$ & $\sim 0$ & $\sim \alpha_{3}^{6}$ & \\
\hline $\mathcal{Q} \overline{\mathcal{Q}}$ & $1_{S}$ & 1 & 2 & 1 & $9 \alpha_{3}^{2} / 16$ & $\sim \alpha_{3}^{7}$ & $\sim \alpha_{3}^{6}$ & $g g$ \\
\hline $\mathcal{Q} \overline{\mathcal{Q}}$ & $8_{A}$ & 1 & 2 & 1 & $9 \alpha_{3}^{2} / 64$ & $\sim 0$ & $\approx 0.1 \alpha_{3}^{5}$ & \\
\hline $\mathcal{Q \mathcal { Q }}$ & $8_{A}$ & 0 & 2 & 1 & $9 \alpha_{3}^{2} / 64$ & $\sim \alpha_{3}^{7}$ & $\approx 0.1 \alpha_{3}^{5}$ & $(q \bar{q})\left(H H^{*}\right)$ \\
\hline $\mathcal{Q \mathcal { Q }}$ & $8_{S}$ & 0 & 2 & 1 & $9 \alpha_{3}^{2} / 64$ & $\sim 0$ & $\approx 0.1 \alpha_{3}^{5}$ & \\
\hline $\mathcal{Q \mathcal { Q }}$ & $8_{S}$ & 1 & 2 & 1 & $9 \alpha_{3}^{2} / 64$ & $\sim \alpha_{3}^{7}$ & $\approx 0.1 \alpha_{3}^{5}$ & $g g$ \\
\hline $\mathcal{Q Q}$ & $1_{S}$ & 0 & 1 & 0 & $9 \alpha_{3}^{2} / 4$ & 0 & 0 & DM candidate \\
\hline $\mathcal{Q Q}$ & $8_{A}$ & 1 & 1 & 0 & $9 \alpha_{3}^{2} / 16$ & 0 & 0 & \\
\hline $\mathcal{Q Q}$ & $8_{S}$ & 0 & 1 & 0 & $9 \alpha_{3}^{2} / 16$ & 0 & 0 & \\
\hline $\mathcal{Q Q}$ & $1_{S}$ & 0 & 2 & 0 & $9 \alpha_{3}^{2} / 16$ & 0 & $\sim \alpha_{3}^{6}$ & \\
\hline $\mathcal{Q Q}$ & $8_{A}$ & 1 & 2 & 0 & $9 \alpha_{3}^{2} / 64$ & 0 & $\sim \alpha_{3}^{6}$ & \\
\hline $\mathcal{Q Q}$ & $8_{S}$ & 0 & 2 & 0 & $9 \alpha_{3}^{2} / 64$ & 0 & $\sim \alpha_{3}^{6}$ & \\
\hline $\mathcal{Q Q}$ & $1_{S}$ & 1 & 2 & 1 & $9 \alpha_{3}^{2} / 16$ & 0 & $\sim \alpha_{3}^{6}$ & \\
\hline $\mathcal{Q Q}$ & $8_{A}$ & 0 & 2 & 1 & $9 \alpha_{3}^{2} / 64$ & 0 & $\approx 0.1 \alpha_{3}^{5}$ & \\
\hline $\mathcal{Q Q}$ & $8_{S}$ & 1 & 2 & 1 & $9 \alpha_{3}^{2} / 64$ & 0 & $\approx 0.1 \alpha_{3}^{5}$ & \\
\hline
\end{tabular}

$$
\left\{\begin{array}{l}
Y_{\mathcal{Q}}^{-1}(z) \approx Y_{\mathcal{Q}}^{-1}\left(z_{\mathrm{dec}}\right)+\lambda \int_{z_{\mathrm{dec}}}^{z} \frac{d z^{\prime}}{z^{2}}\left[\left\langle\sigma_{\mathrm{ann}}^{\mathrm{eff}} v_{\mathrm{rel}}\right\rangle+\left\langle\sigma_{\mathrm{fall}} v_{\mathrm{rel}}\right\rangle\left(1+\frac{\left\langle\Gamma_{\text {break }}\right\rangle M_{\mathcal{Q}}}{E_{B} H\left(z^{\prime}\right) z^{\prime}}\right)^{-1}\right] \\
Y_{\mathcal{Q Q}}(z) \approx \frac{1}{2}\left[\left(Y_{\mathcal{Q}}^{-1}\left(z_{\mathrm{dec}}\right)+\lambda \int_{z_{\mathrm{dec}}}^{z} \frac{d z^{\prime}}{z^{2}}\left\langle\sigma_{\mathrm{ann}}^{\mathrm{eff}} v_{\mathrm{rel}}\right\rangle\right)^{-1}-Y_{\mathcal{Q}}(z)\right] .
\end{array}\right.
$$

Using the specific rates for the main perturbative bound states listed in Table I we obtain the values of $Y_{\mathcal{Q}}$ and of $Y_{\mathcal{Q Q}}$ at temperatures $T \gg \Lambda_{\mathrm{QCD}}$. The result is shown in Fig. 4(b), where they are denoted as perturbative. We see that such an effect can be neglected. At confinement, nonperturbative QCD effects force all free $\mathcal{Q}$ to bind with SM quarks and gluons to form strongly interacting hadrons, as discussed in the following.

\section{B. $\mathcal{Q}$ recoupling at $T \gtrsim \Lambda_{\mathrm{QCD}}$}

DM annihilations recouple below the decoupling temperature $T_{\mathrm{dec}}$ if the thermally averaged DM annihilation cross section $\sigma_{\text {ann }}(T)$ grows at low temperatures faster than $1 / T$. In such a case DM recouples, and its abundance $n_{\mathrm{DM}}$ is further reduced. A tree-level cross section $\sigma_{\text {ann }} \sim g^{4} / M_{\mathrm{DM}}^{2}$ does not recouple. A Sommerfeld enhancement $S \sim 1 / v_{\text {rel }} \propto 1 / \sqrt{T}$ 


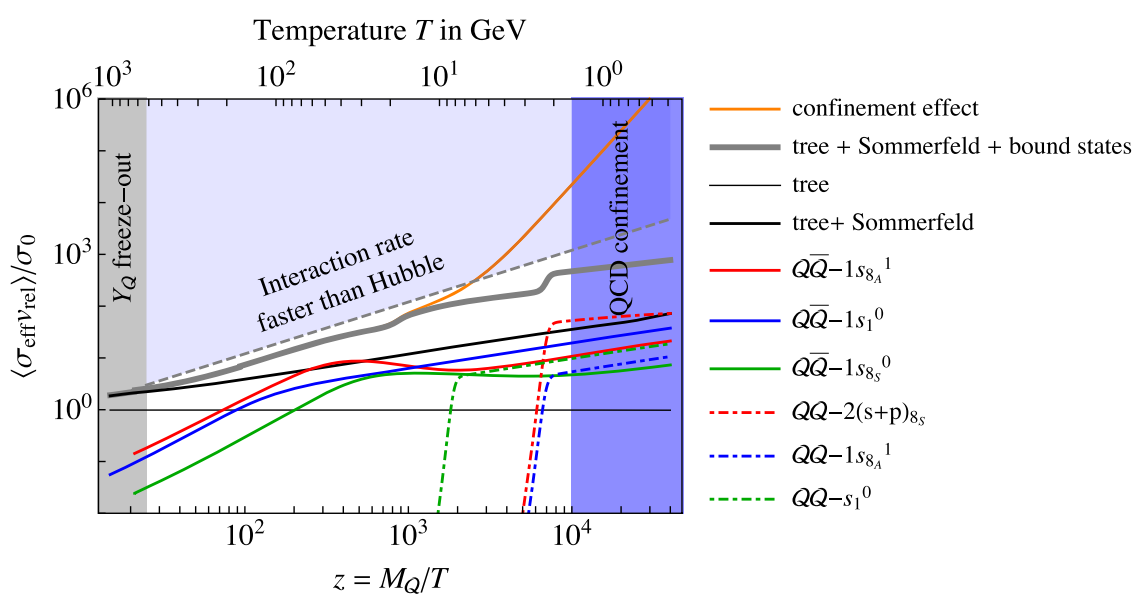

FIG. 3. Thermally averaged effective annihilation cross section in units of $\sigma_{0}=\pi \alpha_{3}^{2} / M_{\mathcal{Q}}^{2}$ for $M_{\mathcal{Q}}=12.5 \mathrm{TeV}$. The horizontal line is the tree-level value in $s$ wave; the black curve is the result obtained adding Sommerfeld corrections; the thick gray curve is the result adding also $\mathcal{Q} \overline{\mathcal{Q}}$ bound-state corrections. The other curves show the contributions from the main bound states among those listed in Table I. The orange curve is an estimate of confinement effects that lead to recoupling at low $T \lesssim 10 \mathrm{GeV}$.
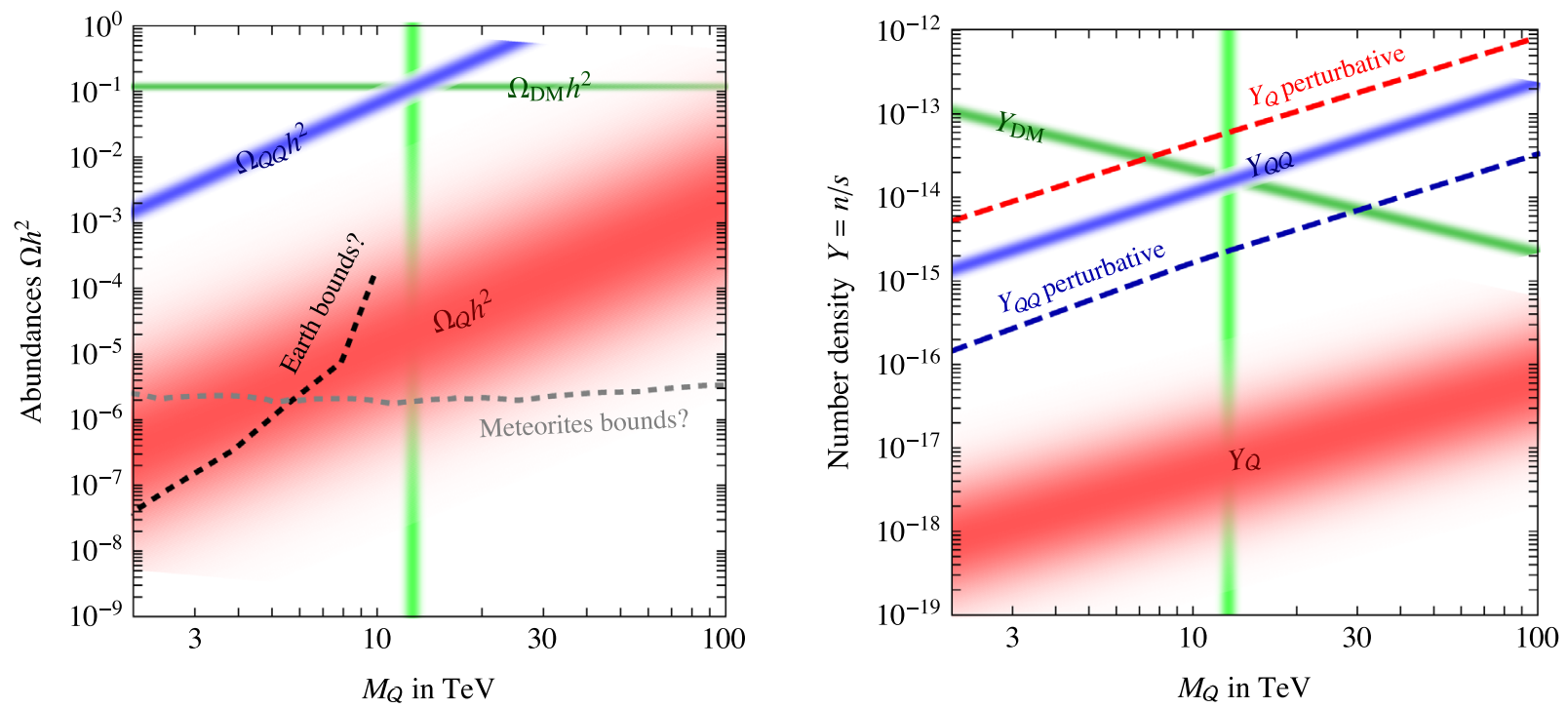

FIG. 4. Thermal relic abundances of the DM $\mathcal{Q} \mathcal{Q}$ hadron (upper) and of hybrid $\mathcal{Q} g$ hadrons (lower band, as obtained varying $\alpha_{\text {eff }}$ and $\sigma_{\mathrm{QCD}} \Lambda_{\mathrm{QCD}}^{2}$ between 1 and $4 \pi$ ). Left: mass densities. The desired DM abundance is reproduced for $M_{\mathcal{Q}} \sim 12.5 \mathrm{TeV}$. The subdominant abundance of hybrid $\mathcal{Q} g$ hadrons and the relative experimental upper bounds are subject to large and undefined nuclear, cosmological and geological uncertainties; see Sec. IV. Right: number densities $Y=n / s$ of $\mathcal{Q Q}$ DM states and of $\mathcal{Q}$ hybrids. We also show the abundance of $\mathcal{Q Q}$ bound states before confinement (dashed curve).

leads to order 1 effects, but not to recoupling (unless enhanced by some resonance). Formation of bound states with small quantum number $n \sim 1$ gives other similar effects. In the previous section we included such order 1 corrections, adapting the results of [16]. ${ }^{5}$ At this stage $\mathcal{Q}$ can form

\footnotetext{
${ }^{5}$ This reference considered neutralino DM in the presence of neutralino/gluino coannihilations. This related scenario is not affected by the new effects at $T \lesssim \Lambda_{\mathrm{QCD}}$ discussed in this paper as long as the gluino/neutralino mass difference is larger than $\Lambda_{\mathrm{QCD}}$. The effects discussed in this paper drastically reduce the cosmological bounds on a long-lived gluino with respect to previous studies [17].
}

relatively deep bound states with heavy quarks, which eventually decay.

The QCD coupling grows nonperturbative at $T \gtrsim \Lambda_{\mathrm{QCD}}$ giving a more dramatic recoupling effect: bound states with binding energy $E_{B n} \sim\left(\alpha_{3} / n\right)^{2} M_{\mathcal{Q}}$ can be formed through a large cross section $\sigma_{\mathrm{ann}} \sim 1 / E_{B n}^{2}$, having omitted powers of the strong coupling. The increase of the cross section as $n \rightarrow \infty$ is tamed by a competing effect: only bound states with $E_{B n} \gtrsim T$ are actually formed at temperature $T$ (as better discussed in Appendix B), leading to a recoupling cross section that grows as $\sigma_{\mathrm{ann}} \sim 1 / T^{2}$ for $T \gtrsim \Lambda_{\mathrm{QCD}}$. 


\section{Chromodark synthesis at $T \sim \Lambda_{\mathrm{QCD}}$}

This effect culminates after confinement. Cosmological effects of confinement begin when the Coulombian force $\alpha_{\text {eff }} / r^{2}$ becomes weaker than the string tension $\sigma(T)$ at the typical distance $r \sim 1 / T$. Given that gluons and quarks are much more abundant than $\mathcal{Q}$, the free $\mathcal{Q}$ form $\mathcal{Q} g$ and $\mathcal{Q} q \bar{q}^{\prime}$ bound states, which have a binding energy of order $\Lambda_{\mathrm{QCD}}$ and scatter among themselves and with other hadrons with cross sections of typical QCD size, $\sigma_{\mathrm{QCD}}=c / \Lambda_{\mathrm{QCD}}^{2} \quad$ with $c \sim 1$. In this stage $H \sim \Lambda_{\mathrm{QCD}}^{2} / M_{\mathrm{Pl}} \sim 10^{-20} \Lambda_{\mathrm{QCD}}$, such that a $\mathcal{Q} g$ hadron experiences $10^{20} \mathrm{QCD}$ scatterings. Given that the relative abundance of $\mathcal{Q}$ is $Y_{\mathcal{Q}} \sim 10^{-14}$, two $\mathcal{Q} g$ meet, forming either deep $\mathcal{Q} \mathcal{Q}$ hadrons (which remain as DM) or $\mathcal{Q} \overline{\mathcal{Q}}$ hadrons (which annihilate into SM particles). The abundance of $\mathcal{Q}$-only hadrons gets dramatically suppressed, until they decouple.

While most DM particles form in this phase, a precise description is not needed to compute the final abundances, which are dominantly determined by what happens during the final redecoupling, where the dominant SM degrees of freedom are semirelativistic pions, while the baryon abundance is negligible, in view of the Boltzmann factor $e^{-m_{p} / T}$ and of the small asymmetry.

\section{D. $\mathcal{Q}$ redecoupling at $T \lesssim \Lambda_{\mathrm{QCD}}$}

We need a precise description of the final redecoupling which occurs at temperatures of tens of $\mathrm{MeV}$. One might think that the simplified Boltzmann equations for the density of free $\mathcal{Q}$ and of $\mathcal{Q Q}$ bound states, Eq. (14), can be replaced with corresponding equations for the total density of $B_{\mathcal{Q}}$ bound states $\left(\mathcal{Q} g\right.$ and $\left.\mathcal{Q} q \bar{q}^{\prime}\right)$ and for the total density of $B_{\mathcal{Q Q}}$ bound states.

A slightly different strategy is needed. Indeed, the simplification that allowed us to reduce the network of Boltzmann equations (one for each bound state) to 2 is valid under the following conditions: all $B_{\mathcal{Q}}$ bound states are in thermal equilibrium among them; all $B_{\mathcal{Q Q}}$ bound states are in thermal equilibrium among them. Bound states are subject to QCD interactions, with large $\sigma_{\mathrm{QCD}}$ cross sections, such that the corresponding interaction rates are much faster than the Hubble rate. However, as discussed in Sec. II C, nonperturbative QCD interactions now lead to the formation of a large variety of bound states, with large $n$ and $\ell$ quantum numbers which suppress the decay rates among them. Some decay rates can be slower than the Hubble rate. This issue was solved in Sec. II E where we computed an effective cross section for the formation of all unbreakable $\mathcal{Q Q}$ bound states, which later fall to the $\mathcal{Q Q}$ ground state. The same cross section, almost as large as the QCD cross section, holds for the formation of unbreakable $\mathcal{Q} \overline{\mathcal{Q}}$, which later annihilate,

$$
\sigma_{\text {fall }}=\sigma_{\mathrm{ann}} \lesssim \sigma_{\mathrm{QCD}}
$$

The equality of the classical nonperturbative total cross section for forming $\mathcal{Q} \overline{\mathcal{Q}}$ bound states with the total cross section for forming $\mathcal{Q Q}$ bound states is compatible with the perturbative quantum cross sections computed in Sec. III A. Indeed, because of Fermi antisymmetrization in the $\mathcal{Q} \mathcal{Q}$ case cross sections are twice bigger, while the number of $\mathcal{Q} \overline{\mathcal{Q}}$ states is twice bigger (after restricting to color-singlet bound states and averaging odd with even $\ell$ ).

One extra process can take place: annihilations between $\mathcal{Q} \mathcal{Q}$ and $\overline{\mathcal{Q}} \overline{\mathcal{Q}}$ in their ground states. In Sec. V B we compute its cross section, finding that it can be neglected in our present cosmological context. Together with Eq. (18) this implies a simple result: half of the $\mathcal{Q}$ and $\overline{\mathcal{Q}}$ present before redecoupling annihilate, and half end up in our DM candidates, the $\mathcal{Q} \mathcal{Q}$ and $\overline{\mathcal{Q}} \overline{\mathcal{Q}}$ ground states. Boltzmann equations are only needed to compute how small the residual fraction of $\mathcal{Q}$ is in loose hybrid hadrons, which are phenomenologically relevant in view of their large detection cross sections.

We thereby group bound states in two categories. We define $Y_{\mathcal{Q Q}}$ as the density of all unbreakable $\mathcal{Q} \mathcal{Q}$ bound states, produced with cross section $\sigma_{\text {fall }}$. We define $Y_{\mathcal{Q}}$ as the density of $\mathcal{Q}$ in loose bound states: the $\mathcal{Q}$ in bound states containing a single $\mathcal{Q}\left(\mathcal{Q} g, \mathcal{Q} q \bar{q}^{\prime}\right)$, and those in loose $\mathcal{Q} \mathcal{Q}$ and $\mathcal{Q} \overline{\mathcal{Q}}$ bound states at relative distances $\sim 1 / \Lambda_{\mathrm{QCD}}$, which get broken by QCD scatterings.

The relevant Boltzmann equation is

$$
\begin{aligned}
s H z \frac{d Y_{\mathcal{Q}}}{d z} & =-2\left(\gamma_{\text {fall }}^{\text {eff }}+\gamma_{\text {ann }}^{\text {eff }}\right)\left[\frac{Y_{\mathcal{Q}}^{2}}{Y_{\mathcal{Q}}^{\mathrm{eq} 2}}-1\right], \\
s H z \frac{d Y_{\mathcal{Q}}}{d z} & =\gamma_{\text {fall }}^{\text {eff }}\left[\frac{Y_{\mathcal{Q}}^{2}}{Y_{\mathcal{Q}}^{\mathrm{eq} 2}}-1\right],
\end{aligned}
$$

valid for $T \lesssim \Lambda_{\mathrm{QCD}}$, i.e., $z \gtrsim z_{\mathrm{QCD}} \equiv M_{\mathcal{Q}} / \Lambda_{\mathrm{QCD}}$. In the nonrelativistic limit the space-time density of interactions is determined by the cross sections as $2 \gamma \simeq\left(n_{B_{\mathcal{Q}}}^{\mathrm{eq}}\right)^{2}\left\langle\sigma v_{\text {rel }}\right\rangle$. The asymptotic solutions to this system of equations are

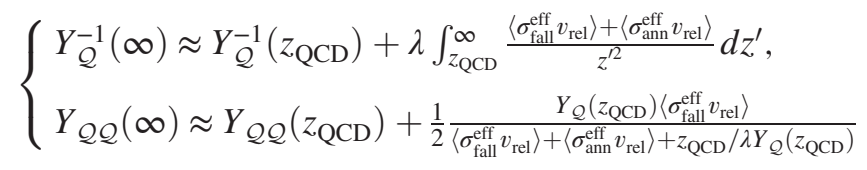

with the last term roughly equaling $Y_{\mathcal{Q}}\left(z_{\mathrm{QCD}}\right) / 4$. Figure 4 shows our final result: the DM abundance and the hybrid abundance as a function of the only free parameter, $M_{\mathcal{Q}}$. The left panel shows the mass abundances $\Omega=\rho / \rho_{\text {cr }}$; the right panel shows the number abundances $Y=n / s$. The hybrid abundances are plotted as bands, given that they are affected by QCD uncertainties; smaller values are obtained 
Temperature $T$ in $\mathrm{GeV}$

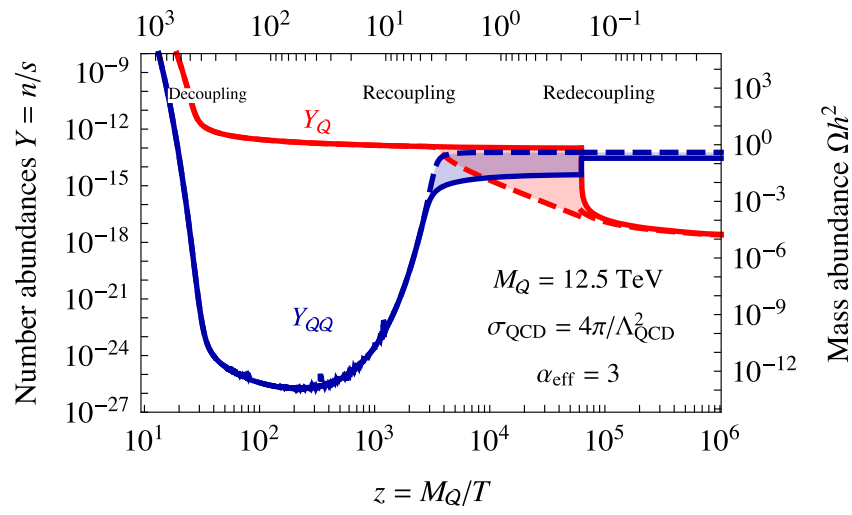

FIG. 5. Cosmological evolution of the abundances of $\mathcal{Q}$ states and of $\mathcal{Q} \mathcal{Q} \mathrm{DM}$ states for $M_{\mathcal{Q}}=12.5 \mathrm{TeV}$. The uncertain phase at $T \gtrsim \Lambda_{\mathrm{QCD}}$ negligibly affects the final relic abundances: the dashed curves assume nonperturbative effects before confinement estimated as $\sigma=\sigma_{\mathrm{QCD}}\left(\Lambda_{\mathrm{QCD}} / T\right)^{2}$; the solid curves neglect such effects. The mass abundance on the right axis is computed assuming $\mathcal{Q} \mathcal{Q}$ particles with mass $2 M_{\mathcal{Q}}$.

for larger $c=\sigma_{\mathrm{QCD}} \Lambda_{\mathrm{QCD}}^{2}$ and for larger $\alpha_{\mathrm{eff}}$. Varying them between 1 and $4 \pi$, the hybrid abundance changes by a factor 100. The DM abundance, less affected by QCD uncertainties, is plotted as a blue curve. The right panel shows that the DM $\mathcal{Q} \mathcal{Q}$ abundance is mostly made at nonperturbative level; the perturbative bound states computed in Sec. III A only play a significant role in enhancing $\mathcal{Q} \overline{\mathcal{Q}}$ annihilations.

The observed DM abundance is reproduced for

$$
M_{\mathcal{Q}} \approx(12.5 \pm 1) \mathrm{TeV}
$$

and the hybrid mass abundance is about $10^{4}$ smaller than the DM abundance (between $10^{3}$ and $10^{5}$ within our assumed range of QCD parameters). For such a mass, Fig. 5 shows the cosmological evolution of the abundances. It also shows how large uncertainties at $T \sim \Lambda_{\mathrm{QCD}}$ before redecoupling have a negligible impact on the final abundances, which is dominantly determined by redecoupling.

An analytic argument that shows that $\Omega_{\text {hybrid }} \ll \Omega_{\mathrm{DM}}$ is unavoidable and that gives the dependence of the final abundances on $M_{\mathcal{Q}}, M_{\mathrm{Pl}}, \Lambda_{\mathrm{QCD}}$ [Eq. (B4)] is confined to Appendix B because it follows a logic different from the one used in the more accurate numerical computation presented here.

\section{E. Nucleodark synthesis}

Redecoupling is completed at temperatures $T \sim 10 \mathrm{MeV}$. Later nucleons bind into light nuclei at the BBN temperature $T_{\mathrm{BBN}} \sim 0.1 \mathrm{MeV}$. Various authors tried to compute what happens to strongly interacting massive particles (SIMPs) during BBN, and how SIMPs affect ordinary
BBN [18-21]. ${ }^{6}$ Our predicted amount of strongly interacting massive particles, $Y_{\text {SIMP }} \sim 10^{-18}$, has negligible effects on ordinary BBN, which constrains $Y_{\text {SIMP }} \lesssim 10^{-12}$. Such studies however disagree on what happens to SIMPs during BBN. Do SIMPs bind with (some) nuclei? Does a significant fraction of SIMPs remain free?

We present our understanding, but we cannot provide a safe answer. Indeed, nuclear forces are not understood from first principles, not even for ordinary $p$ and $n$ [22]. Longrange nuclear properties are determined by couplings to pions, known thanks to chiral perturbation theory [23]. Heavier QCD states contribute to short-range nuclear forces: however QCD is here only used as inspiration to write phenomenological nuclear potentials to be fitted to $p$, $n$ data; see e.g., [24].

In our scenario there are two types of SIMPs with distinct properties. The $\mathcal{Q} g$ hybrids are an isospin singlet and thereby do not couple to pions. The $\mathcal{Q} q \bar{q}$ hybrids form an isospin triplet (with charges $0, \pm 1$ ) coupled to pions.

Presumably $\mathcal{Q} q \bar{q}^{\prime}$ are heavier and decay promptly to $\mathcal{Q} g$. Then, the $\mathcal{Q} g$ singlet states, which do not feel the pion force, are expected to behave similarly to the $\Lambda$ baryon, which does not bind to protons to form heavy deuterons [25]. Maybe such SIMPs do not bind with any nuclei, or maybe they find a way to form bound states with big enough nuclei. An attractive force can be provided by exchange of an isospon-singlet scalar meson, such as the $\sigma$ (mass $M \sim 0.6 \mathrm{GeV}$ ) or glueballs (mass $M \sim 1.5 \mathrm{GeV}$ ) provided that their effective Yukawa couplings $y_{\text {SIMP }}$ and $y_{N}$ to the SIMP and to nucleons are large enough and have the same sign. In spherical well and Born approximation and for $M_{\mathcal{Q}} \gg M$, the hybrid can form a bound state in a nucleus with atomic number $A$ if [26]

$$
y_{\mathrm{SIMP}} y_{N}>\frac{12 \pi}{A^{5 / 3}} \frac{M^{2}}{\mathrm{GeV}^{2}} \text {. }
$$

If SIMPs bind to light nuclei, after BBN they dominantly end up in Helium or free, with a relatively large amount in Beryllium, according to $[19,20]$.

The $\mathcal{Q} q \bar{q}^{\prime}$ states, which feel the pion force, have an interaction potential of approximately $2 \mathrm{fm}$. If they are the lighter stable bound states, during BBN they get incorporated into nuclei with an efficiency close to $100 \%$ [21]. In the Milky Way, SIMPs in charged nuclei can lose a significant fraction of their energy by interactions with ambient matter.

No SIMP searches have yet been performed in galactic clouds, which would probe the SIMP primordial abundance. After BBN, SM matter forms stars and planets: primordial SIMPs sink to their center before these objects possibly solidify. Stars (rather than BBN) later produce the

\footnotetext{
${ }^{6}$ Here and in the following, by SIMP we mean particles that interact strongly with SM particles.
} 
observed elements heavier than He. In the next section we estimate the present geological abundance of SIMPs.

\section{SIGNALS OF RELIC HYBRID HADRON}

In our model $\mathcal{Q}$-onlyum DM is accompanied by hybrid hadrons, containing heavy colored $\mathcal{Q}$ bound together with SM quarks or gluons. In this section we discuss their signals. While SIMP DM has been excluded long ago, in our model SIMPs have a subdominant abundance, $f_{\text {SIMP }} \equiv$ $\rho_{\text {SIMP }} / \rho_{\text {DM }}$ below $10^{-3}$, possibly a few orders of magnitude smaller. Such a small value of $\rho_{\text {SIMP }}$ makes indirect SIMP detection signals negligible $\left(f_{\mathrm{SIMP}}^{2} \sigma_{\mathrm{QCD}} \lesssim 10^{-24} \mathrm{~cm}^{3} / \mathrm{sec}\right)$ despite the fact SIMPs interact with matter nucleons and with themselves through large cross sections of order $\sigma_{\mathrm{QCD}} \sim 1 / \Lambda_{\mathrm{QCD}}^{2}$. See also [27]. In some models SIMPs can have electric charge (fractional in exotic models).

As discussed in Sec. IV A, galactic SIMPs are stopped by the upper atmosphere of the Earth and slowly sink. Thereby SIMPs are not visible in direct detection experiments performed underground. Their later behavior depends on whether SIMPs bind with nuclei: if yes they indirectly feel atomic forces; otherwise they sink even within solid bodies, such as the present Earth. In Sec. IV B we summarize bounds on the SIMP abundance, to be compared with their present abundance, estimated in Secs. IV C and IV D.

\section{A. Direct detection of hybrid hadrons}

Despite their reduced abundance, SIMPs would be excluded by a dozen orders of magnitude, if they reached the underground direct detection detectors with enough energy to trigger events. This is not the case. The energy loss of a neutral SIMP in matter is [28]

$$
\frac{d E}{d x}=-E \sum_{A} n_{A} \sigma_{A} \frac{2 m_{A}}{M_{\mathcal{Q}}} \quad \text { for } m_{A} \ll M_{\mathcal{Q}},
$$

where $n_{A}$ is the number density of nuclei with atomic number $A$ and mass $m_{A} \approx A m_{p} ; 2 m_{A} / M_{\mathcal{Q}}$ is the fractional energy loss per collision and $\sigma_{A} \approx \sigma_{p} A^{2}\left(m_{A} / m_{p}\right)^{2}$ is the SIMP cross section on a nucleus [29], written in terms of the SIMP scattering cross section on protons, $\sigma_{p} \approx$ $\pi / \Lambda_{\mathrm{QCD}}^{2} \approx 1.610^{-26} \mathrm{~cm}^{2}$. The cross section $\sigma_{A}$ is coherently enhanced at the energies of interest for us, $E=$ $M_{\mathcal{Q}} v^{2} / 2 \sim \mathrm{MeV}$ for $v \sim 10^{-3}$. The densities $n_{A}$ in the Earth's crust can be written as $n_{A}=f_{A} \rho / m_{A}$ where $\rho$ is the total mass density and $f_{A}$ is the mass fraction of material $A, \sum_{A} f_{A}=1$. The energy loss following from Eq. (23) is

$$
E(x)=E_{0} \exp \left[-\int \rho d x \frac{\mathrm{m}^{2}}{70 \mathrm{~kg}} \frac{\left\langle A^{4}\right\rangle}{16.6^{4}} \frac{10 \mathrm{TeV}}{M_{\mathcal{Q}}} \frac{\sigma_{p}}{\pi / \Lambda_{\mathrm{QCD}}^{2}}\right] .
$$

Thereby SIMPs with $M_{\mathcal{Q}} \approx 10 \mathrm{TeV}$ thermalize in the Earth's atmosphere, which has a column depth of $10^{4} \mathrm{~kg} / \mathrm{m}^{2}$ and $\left\langle A^{4}\right\rangle^{1 / 4} \approx 16.6$, before reaching the crust with $\left\langle A^{4}\right\rangle^{1 / 4} \approx 31$ and density $\rho \approx 3 \mathrm{~g} / \mathrm{cm}^{3}$. SIMPs do not reach direct detection experiments, situated about a $\mathrm{km}$ underground.

Some direct detection searches have been performed by balloon experiments at high altitudes. The authors of [30] claim that it is questionable whether such experiments exclude a SIMP with density $\rho_{\text {SIMP }}=\rho_{\text {DM }}$. Our predicted abundance $\rho_{\text {SIMP }} \sim 10^{-4} \rho_{\text {DM }}$ is allowed.

After thermalization, SIMPs diffuse with thermal velocity $v_{\text {thermal }} \approx \sqrt{6 T / M_{\mathcal{Q}}} \approx 40 \mathrm{~m} / \mathrm{s}$ at temperature $T \approx 300 \mathrm{~K}$. In the Earth's gravitational field $g=9.8 \mathrm{~m} / \mathrm{s}^{2}$, SIMPs not bound to nuclei sink with a small drift velocity that can be estimated as follows. Each collision randomizes the SIMP velocity because $v_{\text {drift }} \ll v_{\text {thermal }}$. Thereby the drift velocity is the velocity $v_{\text {drift }} \approx g \tau / 2$ acquired during the time $\tau \approx d /$ $v_{\text {thermal }}$ between two scatterings, where $d=1 /\left(\sum_{A} n_{A} \sigma_{A}\right) \sim$ $0.1 \mathrm{~mm}$ in the Earth's crust. Thereby the sinking velocity is

$$
v_{\text {drift }} \approx 0.1 \mathrm{~km} / \mathrm{yr} \text {. }
$$

Diffusion gives a nonuniform SIMP density on the length scale $T / M_{\mathcal{Q}} g \approx 25 \mathrm{~m}$ dictated by the Boltzmann factor $e^{-M_{\mathcal{Q}} g h / T}$.

Finally, SIMPs concentrate around the center of the Earth, where they annihilate heating of the Earth [30]. Bounds on such an effect imply that the SIMP abundance must be subdominant with respect to the DM abundance, $\rho_{\text {SIMP }}<10^{-3} \rho_{\text {DM }}$. This bound is satisfied in our model, where $\rho_{\text {SIMP }} \sim 10^{-4} \rho_{\text {DM }}$.

The situation is somehow different if SIMPs bind with (some) nuclei, either during BBN (mostly forming He), or by colliding with nuclei in the Earth's atmosphere (possibly mostly forming $\mathrm{N}, \mathrm{O}, \mathrm{He}, \mathrm{H}$ ) or crust. A SIMP contained in a hybrid nucleus with charge $z \sim 1$ has a much bigger energy loss in matter, as computed by Bethe,

$\frac{d E}{d x} \approx \frac{K z^{2}}{\beta^{2}} \ln \frac{2 m_{e} \beta^{2}}{I}, \quad K=\frac{4 \pi \alpha^{2} n_{e}}{m_{e}}, \quad I \sim Z 10 \mathrm{eV}$.

The mean free path in Earth of a SIMP in a charged state is thereby $L_{ \pm} \sim M_{\mathcal{Q}} \beta^{4} / K \sim 210^{-5} \mathrm{~cm}(\beta / 0.001)^{4}$. Again, SIMPs do not reach underground detectors. The main difference is that SIMPs bound in nuclei sink in the ocean and in the primordial Earth, but not in the solid crust, where electric atomic forces keep their positions fixed on geological time scales.

\section{B. Searches for accumulated hybrid hadrons}

Experimental searches for accumulated SIMPs consist in taking a sample of matter, and searching if some atom has 
an anomalous mass or charge; see [31] for a recent review. The results, detailed below, imply relative abundances smaller than $\mathcal{O}\left(1 / N_{A}\right)$ (inverse of the Avogadro number) in the selected samples.

The searches often involve a first phase of sample enrichment in hybrids (for example centrifuge treatment of a sample of water, or use of radioactive materials), followed by a second phase of hybrid detection, with the most successful being the mass spectroscopy and Rutherford backscattering [32].

Limits on the SIMP fraction in the sample depend on the SIMP mass: in the range $\mathrm{GeV}$ to $\mathrm{TeV}$ best bounds are derived from mass spectroscopy of enriched seawater samples [33]. Here the hypothetical particle is a positively charged SIMP, which could form heavy water replacing a proton. The bounds on the relative abundance are of order $N_{\text {SIMP }^{+}} / N_{N}<10^{-27}$ where $N_{N}$ is the number of nuclei.

For heavier SIMPs, mass spectroscopy seems to provide weaker limits. The stringent limit stems from studies of material from meteorites. In [32] the Rutherford backscattering technique was used to set a limit on the SIMP-tonucleon number density in the tested meteorites that covers the range $100 \mathrm{GeV}<M_{\mathrm{SIMP}}<10^{7} \mathrm{GeV}$. This technique does not depend on the SIMP charge and thus also applies to neutral SIMPs. For $M_{\text {SIMP }} \sim 10 \mathrm{TeV}$ the limit is [32]

$$
\frac{N_{\text {SIMP }}}{N_{n}} \lesssim 310^{-14} \frac{10 \mathrm{TeV}}{M_{\text {SIMP }}} \quad \text { (meteorites) }
$$

where $N_{n}$ is the number of nucleons.

These bounds should be compared with the predicted SIMP abundance in the selected samples. If the tested samples were representative of the average cosmological composition, our model would predict

$\left.\frac{N_{\mathrm{SIMP}}}{N_{n}}\right|_{\text {cosmo }}=\frac{m_{N}}{M_{\mathcal{Q}}} \frac{\Omega_{\mathrm{SIMP}}}{\Omega_{b}}=510^{-9} \frac{10 \mathrm{TeV}}{M_{\mathcal{Q}}} \frac{f_{\mathrm{SIMP}}}{10^{-5}}$,

having used the cosmological density of baryonic matter, $\Omega_{b} h^{2} \approx 0.022$, and of DM, $\Omega_{\mathrm{DM}} h^{2} \approx 0.12$. The predicted abundance in the selected samples is much lower than the cosmological average and depends on their geological history.

\section{Abundance of hybrid hadrons in the Earth}

Testing a sample of sea water does not lead to bounds, because the atoms that contain heavy hybrid hadrons sink to the bottom. Similarly, the Earth once was liquid, so that the primordial heavy hybrids sank to the core of the Earth. ${ }^{7}$

\footnotetext{
${ }^{7}$ The Earth's crust contains significant abundances of some heavier elements: those that preferentially form chemical bounds with light elements, reducing the average density. This possibility does not hold for too heavy hybrids with mass $\sim 10 \mathrm{TeV}$.
}

Objects made of normal matter accumulate SIMPs due to collisions with SIMP relics in the interstellar medium. Heavy hybrids accumulated in the Earth's crust, if captured by nuclei, presumably stopped sinking after which the crust solidified. In order to set bounds, we thereby consider the SIMPs captured by the Earth in the time $\Delta t \sim 4$ Gyr passed since it is geologically quasistable. We ignore convective geological motion. The Earth is big enough to stop all SIMPs, so that the total mass of accumulated SIMPs is

$$
M \sim \rho_{\mathrm{SIMP}} v_{\text {rel }} \pi R_{E}^{2} \Delta t \sim 2.510^{10} \mathrm{~kg} \frac{f_{\text {SIMP }}}{10^{-5}}
$$

having inserted the escape velocity from the Galaxy $v \sim 10^{-3}$ and assuming that the SIMP galactic density follows the DM matter halo density $\rho_{\mathrm{DM}} \approx 0.3 \mathrm{GeV} / \mathrm{cm}^{3}$ as $n_{\mathrm{SIMP}}=f_{\mathrm{SIMP}} \rho_{\mathrm{DM}} / M_{\mathrm{SIMP}}$. The rate of $\mathcal{Q} \overline{\mathcal{Q}}$ annihilations of stopped SIMPs is negligible, because suppressed by $e^{-M_{\mathcal{Q}} r}$ where $r$ is the macroscopic distance between $\mathcal{Q}$ and $\overline{\mathcal{Q}}{ }^{8}$

The number of SIMPs accumulated in the Earth is

$\left.\frac{N_{\text {SIMP }}}{N_{n}}\right|_{\text {Earth }}=\frac{M}{M_{\mathcal{Q}}} \frac{m_{N}}{M_{\text {Earth }}} \approx 410^{-19} \frac{10 \mathrm{TeV}}{M_{\mathcal{Q}}} \frac{f_{\text {SIMP }}}{10^{-5}} \frac{v_{\text {rel }}}{10^{-3}}$.

If SIMPs are not captured by nuclei and sink as in Eq. (25), their present density in the crust is negligibly small, $N_{\text {SIMP }} / N_{n} \sim 10^{-23}$. If SIMPs get captured in nuclei, a significant fraction of such SIMPs could be in the crust, with a local number density higher by some orders of magnitude. In Fig. 4 we plot the bound from Earth searches assuming that all SIMPs stop in the atmosphere and sink slowly through Earth until captured by a nucleus, which might happen in the upper $10 \mathrm{~km}$. The capture cross section with nuclei is discussed below.

\section{Abundance of hybrid hadrons in meteorites}

Meteorites result from accumulation of interstellar dust and contain heavy elements. The tested meteorites consist mainly of carbon and/or iron. These elements have not been produced by big bang nucleosynthesis, which produced $\mathrm{H}$ and $\mathrm{He}(Z \leq 2)$, or by cosmic ray fission, which produced $\mathrm{Li}, \mathrm{Be}, \mathrm{B}(Z \leq 5)$. Heavier elements have been synthesized from nuclear burning in stars and have later been dispersed away through various explosive processes: core-collapse supernovæ, accretion supernovæ, merging neutron stars and $r$-process nucleosynthesis. Primordial SIMPs would have sunk to the center of stars, and would have presumably remained trapped there, undergoing $\mathcal{Q} \overline{\mathcal{Q}}$ annihilations.

\footnotetext{
${ }^{8}$ The SIMP thermonuclear energy content $M c^{2}$ could be artificially released through $\mathcal{Q} \overline{\mathcal{Q}}$ annihilations, and is about $10^{4}$ times larger than the world fossil energy reserve, $10^{23} \mathrm{~J}$.
} 
Thereby, the SIMP relative abundance in meteorites made of heavy elements is expected to be significantly smaller than the average relative cosmological abundance.

In order to set bounds we compute the amount of SIMPs accumulated in meteorites. Meteorites are the oldest objects in the Solar System and are so small that heavy hybrids do not sink in them. While the Earth is large enough that it captures all SIMPs intercepted by its surface, we consider meteorites small enough that the opposite limit applies: SIMPs are captured by all nuclei within the volume of the meteorite. Thus we need to estimate the probability $\wp$ that a nucleus captured a SIMP in a time $\Delta t$,

$$
\begin{aligned}
\left.\frac{N_{\text {SIMP }}}{N_{n}}\right|_{\text {meteorite }} & =\wp=n_{\text {SIMP }} \sigma_{\text {capture }} v_{\text {rel }} \Delta t \\
& \approx 710^{-12} \frac{\sigma_{\text {capture }}}{1 / \Lambda_{\text {QCD }}^{2}} \frac{10 \mathrm{TeV}}{M_{\text {SIMP }}} \frac{f_{\text {SIMP }} \frac{\Delta t}{10^{-5}} \frac{v_{\text {rel }}}{5 \mathrm{Gyr}} 10^{-3}}{}
\end{aligned}
$$

This value is roughly 2 orders of magnitude above the meteorite bound in Eq. (27).

However, the capture cross sections of SIMP by nuclei are very uncertain. Taking into account that they are not coherently enhanced, the maximal value is the area of the nucleus, $\sigma_{\text {capture }} \sim A^{2 / 3} / \Lambda_{\mathrm{QCD}}^{2}$ [34]. The measured capture cross sections of neutrons by nuclei are smaller: in most cases $\sigma_{\text {capture }} \sim 0.01 / \Lambda_{\mathrm{QCD}}^{2}$ at $\mathrm{MeV}$ energies. Assuming this capture cross section we obtain the possible meteorite bound

$$
f_{\mathrm{SIMP}}=\frac{\rho_{\mathrm{SIMP}}}{\rho_{\mathrm{DM}}} \lesssim 10^{-5} \frac{\sigma_{\text {capture }}}{0.01 / \Lambda_{\mathrm{QCD}}^{2}}
$$

plotted in Fig. 4 and summarized in Table II. Our SIMPs have $\mathrm{MeV}$ energies, but the long-distance attractive force mediated by pions (present for neutrons, where it is the only effect understood from first principles) is absent for $\mathcal{Q} g$ SIMPs, which are isospin singlets. Their capture cross

TABLE II. Experimental bounds on the density of strongly interacting massive particles with nonexotic electric charges, compared to the expected abundance of our hybrid, roughly estimated assuming that it binds in nuclei (otherwise they sink), and assuming $f_{\text {SIMP }} \approx 10^{-5}$.

\begin{tabular}{lccc}
\hline \hline \multirow{2}{*}{$\begin{array}{l}\text { Element } \\
\text { studied }\end{array}$} & $N_{\text {SIMP }} / N_{N}$ at $M_{\text {SIMP }}=10 \mathrm{TeV}$ & Formation \\
\cline { 2 - 3 } & Bound & Expectation? & mechanism \\
\hline He space & $\ldots$ & $10^{-10}$ & BBN \\
Be Earth & $710^{-9}[35]$ & No & BBN \\
Oxygen water & $310^{-14}[35]$ & No & Accumulation \\
Enriched petro-C & $10^{-16}[35]$ & $10^{-15} ?$ & Accumulation \\
Iron Earth & $10^{-12}[36]$ & $10^{-15}$ ? & Accumulation \\
Meteorites & $410^{-14}[32]$ & $10^{-14}$ ? & Capture \\
\hline \hline
\end{tabular}

section could be much smaller, and possibly our SIMPs do not form bound states with nuclei, such that meteorite bounds are not applicable.

\section{DARK MATTER SIGNALS}

In our model $\mathrm{DM}$ is a $\mathcal{Q Q}$ hadron. In this section we discuss the DM signals: direct detection (Sec. VA), indirect detection (Sec. V B) and collider (Sec. V C).

\section{A. Direct detection of DM}

Direct detection of DM is a low-energy process, conveniently described through effective operators. Composite DM gives operators which can be unusual with respect to those characteristic of elementary DM with tree-levelmediated interactions to matter. For example, a fermionic bound state can have a magnetic dipole moment, which is strongly constrained. In our case DM is a nonrelativistic scalar bound state $\mathcal{Q} \mathcal{Q}$ made of two colored neutral fermions $\mathcal{Q}$. Its dominant interaction with low-energy gluons is analogous to the Rayleigh scattering of photons from neutral hydrogen. Describing our $\mathcal{Q Q}$ bound state as a relativistic field $B$ with canonical dimension 1, the effective Lagrangian is

$\mathcal{L}_{\text {eff }}=C_{S}^{g} \mathcal{O}_{S}^{g}+C_{T_{2}}^{g} \mathcal{O}_{T_{2}}^{g}=M_{\mathrm{DM}} \bar{B} B\left[c_{E} \vec{E}^{a 2}+c_{B} \vec{B}^{a 2}\right]$

The first expression employs the conventional basis of operators

$$
\begin{aligned}
\mathcal{O}_{S}^{g} & =\frac{\alpha_{3}}{\pi} \bar{B} B\left(G_{\mu \nu}^{a}\right)^{2}, \\
\mathcal{O}_{T_{2}}^{g} & =-\frac{\bar{B} \partial^{\mu} \partial^{\nu} B}{M_{\mathrm{DM}}^{2}} \mathcal{O}_{\mu \nu}^{g} \stackrel{E \ll M_{Q}}{\simeq}-\frac{\bar{B} B}{2}\left[\left(G_{0 i}^{a}\right)^{2}+\left(G_{i j}^{a}\right)^{2}\right],
\end{aligned}
$$

where $\quad\left(G_{\mu \nu}^{a}\right)^{2}=2\left(\vec{B}^{a 2}-\vec{E}^{a 2}\right) \quad$ and $\quad \mathcal{O}_{\mu \nu}^{g} \equiv G_{\mu}^{a \rho} G_{\nu \rho}^{a}-$ $\frac{1}{4} \eta_{\mu \nu} G_{\rho \sigma}^{a} G^{a \rho \sigma}$. In the second expression we rewrote them in terms of the chromoelectric $E_{i}^{a}=G_{0 i}^{a}$ and chromomagnetic $\vec{B}^{a}$ components, such that $c_{E}$ is $4 \pi$ times the chromoelectric polarizability of the bound state, $c_{E} \sim 4 \pi a^{3}$ where $a=2 /\left(3 \alpha_{3} M_{\mathcal{Q}}\right)$ is its Bohr-like radius. Furthermore $c_{B} \ll c_{E}$ is suppressed by the velocity $v \sim \alpha_{3}$ of the $\mathcal{Q}$ in the bound state. Neglecting the chromomagnetic interaction, the coefficients renormalized at the high scale (that we approximate with $M_{Z}$ ) are

$C_{T_{2}}^{g}\left(M_{Z}\right)=-M_{\mathrm{DM}} c_{E}, \quad C_{S}^{g}\left(M_{Z}\right)=\frac{C_{T_{2}}^{g}\left(M_{Z}\right)}{4} \frac{\pi}{\alpha_{3}}$.

The low-energy effective coupling of DM to nucleons is $f_{N}|B|^{2} \bar{N} N$ [37] with 


$$
\frac{f_{N}}{m_{N}}=-12 C_{S}^{g}\left(M_{Z}\right) f_{g}-\frac{3}{4} C_{T_{2}}^{g}\left(M_{Z}\right) g\left(2, M_{Z}\right)
$$

where $f_{g}=0.064$ and $g\left(2, M_{Z}\right)=0.464$. The spinindependent direct detection cross section is

$$
\begin{aligned}
\sigma_{\mathrm{SI}} & =\frac{f_{N}^{2}}{4 \pi} \frac{m_{N}^{2}}{M_{\mathrm{DM}}^{2}} \\
& \approx 2.310^{-45} \mathrm{~cm}^{2} \times\left(\frac{20 \mathrm{TeV}}{M_{\mathrm{DM}}}\right)^{6}\left(\frac{0.1}{\alpha_{3}}\right)^{8}\left(\frac{c_{E}}{1.5 \pi a^{3}}\right)^{2} .
\end{aligned}
$$

This is close to the XeNON1T bound [38], $\sigma_{\mathrm{SI}} \lesssim$ $310^{-44} \mathrm{~cm}^{2} \times M_{\mathrm{DM}} / 20 \mathrm{TeV}$, that holds at $M_{\mathrm{DM}} \gg$ $100 \mathrm{GeV}$ up to the standard assumptions about the DM galactic halo.

Thereby we perform a dedicated computation of the $c_{E}$ coefficient, which is possible in perturbative QCD. Adapting the techniques developed for the hydrogen atom and for bottomonium [39], the effective Lagrangian of Eq. (33) also describes the shift in the $\mathcal{Q} \mathcal{Q}$ ground state energy induced by external chromoelectric and chromomagnetic fields,

$$
H_{\mathrm{eff}}=-\frac{1}{2}\left[c_{E} \vec{E}^{a 2}+c_{B} \vec{B}^{a 2}\right]
$$

The external field $\vec{E}^{a}$ adds a chromodipole interaction to the nonrelativistic Hamiltonian of the $\mathcal{Q Q}$ bound state, as well as the associated non-Abelian effects. Perturbation theory at second order then gives a shift in the ground state energy $E_{10}$, which allows us to reconstruct $c_{E}$ as

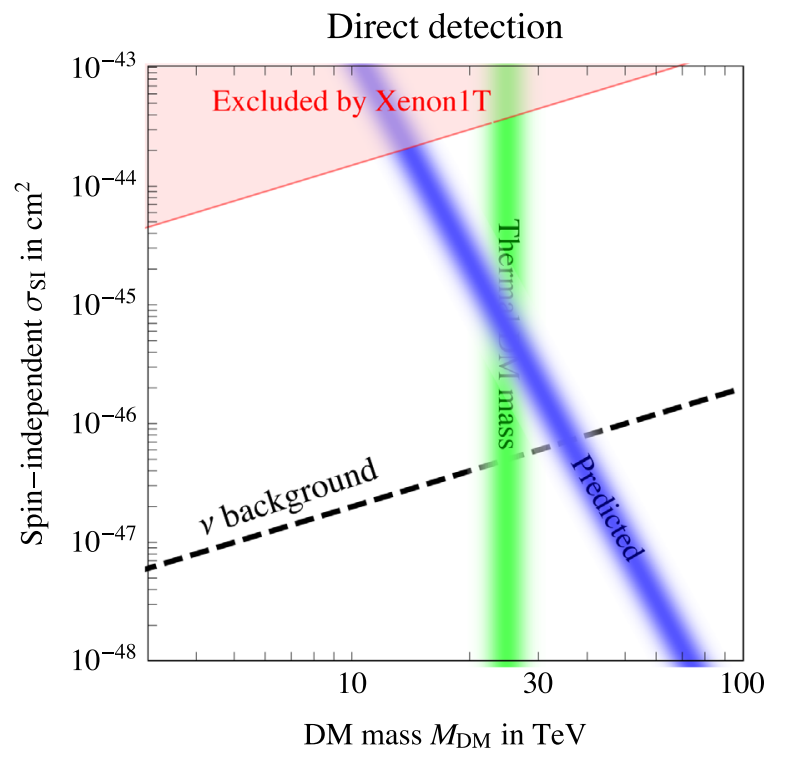

$$
c_{E}=\frac{8 \pi \alpha_{3}}{3} \frac{C}{N_{c}^{2}-1}\left\langle B\left|\vec{r} \frac{1}{H_{8}-E_{10}} \vec{r}\right| B\right\rangle
$$

where $|B\rangle$ is the $\mathcal{Q Q}$ ground state, $N_{c}=3$ and $C$ is the Casimir coefficient, defined by $C \delta_{i j}=\left(T^{a} T^{a}\right)_{i j}$ and equal to 3 for our assumed octet representation. Summing over all allowed intermediate states with free Hamiltonian $H_{8}$ in the octet channel we find (see Appendix C)

$$
\left.c_{E}\right|_{\mathrm{DM}}=(0.36+1.17) \pi a^{3}
$$

where the first (second) contribution arises from intermediate bound (free) states. The non-Abelian nature of QCD manifests in the fact that the allowed intermediate states are $p$-wave color octets: they are less bound (relatively to the ground state) than in the hydrogen atom case, such that our $c_{E}$ coefficient is significantly smaller than what would be suggested by a naive rescaling of the Abelian result.

Equation (40) is the coefficient used as a reference value in the cross section of Eq. (37). Higher order QCD interactions and relativistic effects conservatively amount to a 50\% uncertainty. As plotted in Fig. 6(a) our predicted DM mass $M_{\mathrm{DM}} \approx 25 \mathrm{TeV}$ is higher than the DM mass excluded by direct detection, $M_{\mathrm{DM}} \gtrsim 14 \mathrm{TeV}$.

\section{B. Indirect detection of DM}

Two DM particles in the galactic halo can annihilate into gluons and quarks giving rise to indirect detection signals. The energy spectra of the resulting final-state stable particles $(\bar{p}, \bar{e}, \gamma, \nu)$ are well approximated by the general results of nonrelativistic annihilations computed in [41].

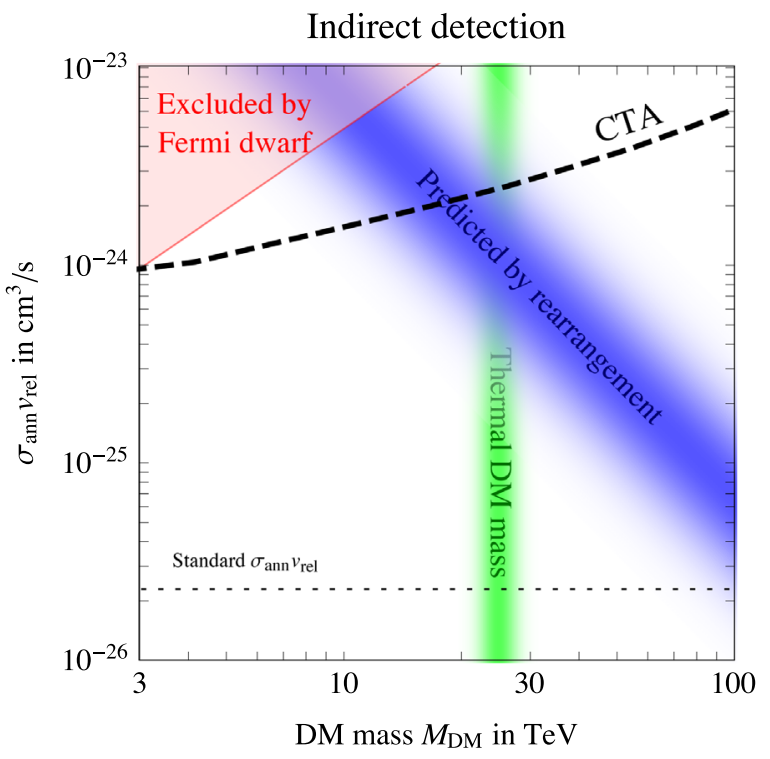

FIG. 6. Left: Direct detection signals of $\mathcal{Q} \mathcal{Q}$ dark matter, as computed in Sec. V A. We also show the neutrino floor, which eventually limits future direct searches. Right: Indirect detection signals as computed in Sec. V B. We show the current dwarf galaxy constraints by FermiLAT, which have only a mild systematic uncertainty due to the dark matter $J$-factor, and the future sensitivity of the CTA [40] experiment to photons from dwarf galaxies. 
We need to compute the annihilation cross section between the $\mathrm{DM}=\mathcal{Q} \mathcal{Q}$ Coloumbian bound state and $\overline{\mathrm{DM}}=\overline{\mathcal{Q}} \overline{\mathcal{Q}}$. It is enhanced and dominated by the recombination process

$$
(\mathcal{Q} \mathcal{Q})+(\overline{\mathcal{Q}} \overline{\mathcal{Q}}) \rightarrow(\mathcal{Q} \overline{\mathcal{Q}})+(\mathcal{Q} \overline{\mathcal{Q}})
$$

followed by later $\mathcal{Q} \overline{\mathcal{Q}}$ annihilations to SM particles. This is similar to what happens for hydrogen/antihydrogen annihilation, which proceeds through recombination $(e p)+(\bar{e} \bar{p}) \rightarrow(e \bar{e})+(p \bar{p})$ followed by later $e \bar{e}$ and $p \bar{p}$ annihilations, giving rise to a large $\sigma_{\text {ann }}$, of atomic-physics size, rather than of particle-physics size, $\sigma_{\mathrm{ann}} \sim \alpha^{2} / m_{e, p}^{2}$.

The DM recombination cross section can be estimated as follows. At relative velocities $v_{\text {rel }}$ comparable to the orbital velocity $\alpha_{3}$ (which corresponds to a center-of-mass kinetic energy $K=M_{\mathcal{Q}} v_{\text {rel }}^{2} / 2$ comparable to the binding energy $E_{B}$ ) the cross section is given by the Bohr radius squared,

$$
\sigma_{\mathrm{ann}} v_{\text {rel }} \sim \pi a^{2} v_{\text {rel }} \sim \frac{1}{\alpha_{3} M_{\mathcal{Q}}^{2}}\left(v_{\text {rel }} \sim \alpha_{3}\right) .
$$

At larger velocities $v_{\text {rel }} \gtrsim \alpha_{3}$ the cross section gets progressively suppressed by $\left(E_{B} / K\right)^{2}$, reducing at $v_{\text {rel }} \sim 1$ to the particle-physics cross section $\sigma_{\mathrm{ann}} \sim \alpha_{3}^{2} / M_{\mathcal{Q}}^{2}$.

At smaller $v_{\text {rel }} \ll \alpha_{3}$ recombination is enhanced by a classical Sommerfeld effect which can be estimated as follows. The interaction between two neutral atoms at distance $r \gg a$ is given by the non-Abelian Van der Waals electric attraction, $V_{\mathrm{el}} \approx-0.7 a^{6} / r^{7}[39,42,43]$, having used Eq. (40) for the numerical coefficient. A four-particle intermediate state forms if $K>\max _{r} V_{\text {eff }}(r)$ where $V_{\text {eff }}=$ $V_{\mathrm{el}}+L^{2} / 2 M_{\mathcal{Q}} r^{2}$ is the usual effective potential. This determines the maximal impact parameter $b_{\max }$, and thereby the cross section ${ }^{9}$

$$
\sigma_{\text {ann }} v_{\text {rel }} \sim \pi b_{\text {max }}^{2} v_{\text {rel }} \sim \frac{v_{\text {rel }}^{3 / 7}}{\alpha_{3}^{12 / 7} M_{\mathcal{Q}}^{2}} \quad\left(\alpha_{3}^{5 / 2} \ll v_{\text {rel }} \ll \alpha_{3}\right) .
$$

At astrophysically low velocities $v_{\text {rel }} \sim 10^{-3} \lesssim \alpha_{3}^{5 / 2}$ the magnetic dipole interaction $V_{\text {mag }} \sim \alpha_{3} / r^{3} M_{\mathcal{Q}}^{2}$ becomes as

\footnotetext{
${ }^{9} \mathrm{~A}$ more precise result can be obtained from a classical computation. Focusing on the color-singlet channel, we numerically compute the classical motion of a $\mathcal{Q} \mathcal{Q}$ bound state in its ground state (circular orbit with radius $a$ in some plane) which collides with relative velocity $v_{\text {rel }}$ and impact parameter $b$ with a similar $\overline{\mathcal{Q}} \overline{\mathcal{Q}}$ system. When the two bound states get closer and interact they can produce two $\mathcal{Q} \overline{\mathcal{Q}}$ bound states, which later annihilate. Confinement takes place at larger distances and plays a negligible role. Averaging over the relative orientations of the two systems and over the impact parameter gives the classical probability for this process, encoded into a velocity dependent cross section.
}

important as the electric interaction, giving $\sigma_{\mathrm{ann}} v_{\mathrm{rel}} \sim$ $\alpha_{3}^{2 / 3} / M_{\mathcal{Q}}^{2} v_{\text {rel }}^{1 / 3}$. Detailed quantum computations suggest that a reasonable estimate consistent with Wigner's threshold law is [44]

$$
\begin{aligned}
\sigma_{\mathrm{ann}} v_{\text {rel }} & \sim \frac{\pi a^{2} v_{\text {rel }} / 2}{\sqrt{E_{\mathrm{kin}} / E_{B}}}=\frac{\sqrt{2} \pi}{3 M_{\mathcal{Q}}^{2} \alpha_{3}} \\
& =1.510^{-24} \frac{\mathrm{cm}^{3}}{\mathrm{sec}} \times\left(\frac{20 \mathrm{TeV}}{M_{\mathrm{DM}}}\right)^{2}\left(\frac{0.1}{\alpha_{3}}\right) .
\end{aligned}
$$

The $1 / \alpha_{3}$ enhancement is numerically mild, given that $\alpha_{3} \sim 0.1$. As a consequence indirect detection signals are below present bounds, as shown in Fig. 6(b). We plotted bounds on gamma ray emission from dwarfs, given that searches in the Galactic center region are subject to large astrophysical uncertainties, and other bounds are weaker.

\section{Collider signals of DM}

While DM usually gives missing-energy signals which are hardly detectable at hadron colliders, DM made of colored quorns $\mathcal{Q}$ gives very visible signals. Indeed, DM constituents $\mathcal{Q}$ are pair produced at colliders via $\mathrm{QCD}$ interactions. After hadronization they form hadrons. Presumably the neutral $\mathcal{Q} g$ is stable, and the charged $\mathcal{Q} q \bar{q}^{\prime}$ are long lived on collider time scales, giving rise to tracks. This is the dream of LHC experimentalists. Experiments at the LHC $p p$ collider at $\sqrt{s}=13 \mathrm{TeV}$ set the bound $M_{\mathcal{Q}} \gtrsim 2 \mathrm{TeV}$ [45]. A larger $\sqrt{s} \sim 85 \mathrm{TeV}$ is needed to discover the quorn with the mass expected from cosmology, $M_{\mathcal{Q}} \sim 12.5 \mathrm{TeV}$. A $p p$ collider with $\sqrt{s}=$ $100 \mathrm{TeV}$ would be sensitive up to $M_{\mathcal{Q}} \lesssim 15 \mathrm{TeV}$ [46], as long as the detector can see the signal.

Furthermore, we explore the possibility of detecting collisions of protons in collider beams with ambient $\mathcal{Q} \mathcal{Q}$ DM. The $\mathcal{Q} \mathcal{Q}$ binding energy is $E_{B} \sim 200 \mathrm{GeV}$. Protons with energies much larger than $E_{B}$ see the $\mathcal{Q Q}$ system as two free $\mathcal{Q}$ and the QCD cross section is suppressed by the energy squared. Protons with energies comparable to $E_{B}$ see the system as a ball with Bohr radius $a=2 / 3 \alpha_{3} M_{\mathcal{Q}}$. The cross section for the excitation of the ground state through the absorption of a gluon can be estimated as the cross section for ionization computed in [16,47]

$$
\sigma=36 \pi^{2} \alpha_{3} a^{2}\left(\frac{E_{B}}{E_{g}}\right)^{4} \frac{1+9 / 4 \zeta^{2}}{1+9 \zeta^{2}} \frac{e^{-6 \zeta \operatorname{arccot}(3 \zeta)}}{1-e^{-3 \pi \zeta}}
$$

where $E_{g}$ is the gluon energy and $\zeta=\alpha_{3} / v_{\text {rel }}=1 /\left(3 a p_{\mathrm{DM}}\right)$ parametrizes the momentum of $\mathcal{Q}$ in the final state. Energy conservation implies $E_{g} \approx E_{B}+M_{\mathrm{DM}} v_{\text {rel }}^{2} / 4$. Figure 7 shows the proton-DM cross section obtained convoluting with parton distribution functions. The event rate in a beam containing $N_{p}$ protons is small, 


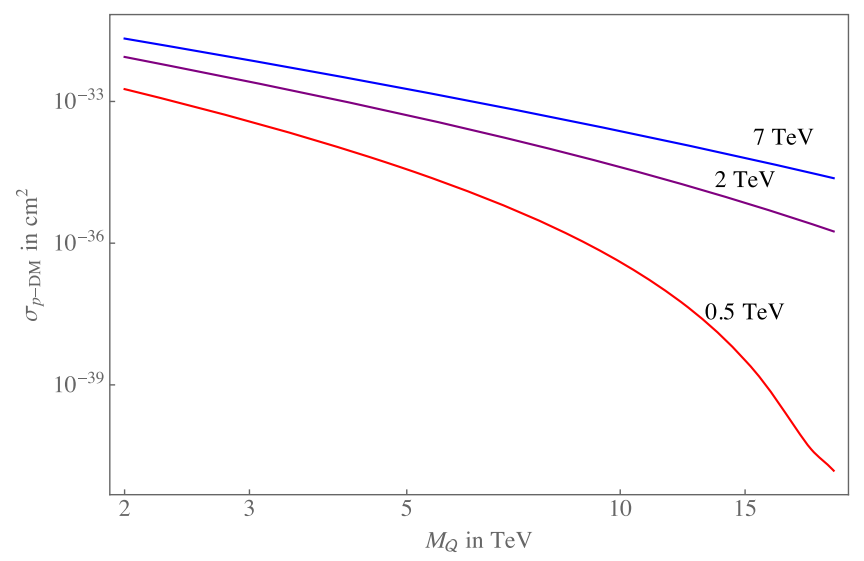

FIG. 7. Cross section for excitation of the $\mathcal{Q} \mathcal{Q}$ DM ground state with a proton beam at 0.5 (red), 2 (purple), and 7 (blue) $\mathrm{TeV}$.

$$
\begin{aligned}
\frac{d N_{p}}{d t} & =N_{p} \sigma \frac{\rho_{\mathrm{DM}}}{2 M_{\mathcal{Q}}} \\
& =\frac{3}{\text { year }} \frac{N_{p}}{10^{20}} \frac{\rho_{\mathrm{DM}}}{0.3 \mathrm{GeV} / \mathrm{cm}^{3}} \frac{20 \mathrm{TeV}}{2 M_{\mathcal{Q}}} \frac{\sigma}{10^{-33} \mathrm{~cm}^{2}} .
\end{aligned}
$$

$\mathcal{Q} \mathcal{Q}$ dark matter excitation by cosmic rays is negligible on cosmological time scales.

\section{CONCLUSIONS}

We have shown that dark matter can be obtained from a colored neutral quark $\mathcal{Q}$ (dubbed quorn) that, after the QCD phase transition, forms deeply bound hadrons made of $\mathcal{Q}$ only (dubbed quorn onlyum), plus traces of hybrid hadrons made of $\mathcal{Q}$ together with SM gluons or quarks (dubbed SIMPs). We explored the simplest model, where $\mathcal{Q}$ is an automatically stable neutral Dirac fermion in the adjoint representation of $\mathrm{SU}(3)_{c}$. Such a state could be a Dirac gluino, or appear in natural axion models (see Sec. II).

Figure 5 shows the cosmological evolution of the DM and hybrid abundances for the value of the quorn mass, $M_{\mathcal{Q}} \approx 12.5 \mathrm{TeV}$, which reproduces the DM cosmological abundance as discussed in Sec. III. A first decoupling occurs, as usual, at $T \sim M_{\mathcal{Q}} / 25$. Quorns recouple while the Universe cools approaching the QCD phase transition at $T \sim \Lambda_{\mathrm{QCD}}$. This opens a phase of chromodark synthesis: quorns fall into $\mathcal{Q} \mathcal{Q}$ singlet bound states, which have a binding energy $E_{B} \sim 200 \mathrm{GeV}$. The cross sections grow large, up to $\sigma_{\mathrm{QCD}} \sim 1 / \Lambda_{\mathrm{QCD}}^{2}$, because excited states with large angular momenta $\ell$ are formed. Such states efficiently cool falling to the ground state before being broken, as computed in Sec. II E where we show that quantum states with $n, \ell \gg 1$ are well approximated by classical physics. It is important to take into account that (non-Abelian) Larmor radiation from elliptic orbits is much larger than for circular orbits.

Details of this uncertain phase are not very important for the final result: one half of free quorns annihilate, and one half end up in $\mathcal{Q Q} \mathrm{DM}$; the small residual abundance of $\mathcal{Q} g$ hybrids, $\rho_{\mathrm{SIMP}} / \rho_{\mathrm{DM}}$ between $10^{-3}$ and $10^{-6}$, is mostly determined at $T \sim 30 \mathrm{MeV}$, when the states decouple again.

In Sec. V we studied DM phenomenology. The quornonlyum DM state $\mathcal{Q} \mathcal{Q}$ with mass $M_{\mathrm{DM}} \approx 2 M_{\mathcal{Q}} \approx 25 \mathrm{TeV}$ has small residual interactions suppressed by powers of $1 / M_{\mathcal{Q}}$. The cross section for direct DM detection is of Rayleigh type, suppressed by $1 / M_{\mathcal{Q}}^{6}$. In Sec. VA we performed a nontrivial QCD bound-state computation, finding a cross section just below present bounds. The cross section for indirect DM detection is enhanced by recombination, $(\mathcal{Q} \mathcal{Q})+(\overline{\mathcal{Q}} \overline{\mathcal{Q}}) \rightarrow(\mathcal{Q} \overline{\mathcal{Q}})+(\mathcal{Q} \overline{\mathcal{Q}})$, and still compatible with bounds (Sec. V B). At colliders quorns manifest as (quasi)stable charged tracks: the LHC sets the bound $M_{\mathcal{Q}} \gtrsim 2 \mathrm{TeV}$.

In Sec. IV we studied the SIMP hybrid states, which have large cross sections of order $1 / \Lambda_{\mathrm{QCD}}^{2}$ and a relic abundance 3 or more orders of magnitude smaller than DM. In view of this, they seem still allowed by the experiments which excluded SIMP DM $\left(\rho_{\text {SIMP }}=\rho_{\text {DM }}\right)$, although a Manhattan-like project would be needed to predict their properties. Our model contains two kind of SIMPs: the isospin-singlet $\mathcal{Q} g$ with no interaction to pions, and the isospin triplet $\mathcal{Q} q \bar{q}^{\prime}$. Presumably the latter are heavier and decay. We do not know whether $\mathcal{Q} g$ can bind with (large enough?) nuclei, and how they would bind during big bang nucleosynthesis, given that there is no first-principle understanding of nuclear potentials. The following statements are safe: our predicted SIMP abundance is so small that they negligibly affect ordinary BBN; SIMPs get stopped by the Earth's atmosphere and are not visible in underground detectors; SIMP annihilations negligibly heat the Earth.

The interpretation of searches for rare hybrid heavy nuclei in samples of materials depends on the history of SIMPs and of the selected samples: from the big bang, to star burning, through Earth geology. The primordial abundance of SIMPs in the Earth and in stars sank down to their centers, undergoing $\mathcal{Q} \overline{\mathcal{Q}}$ annihilations. Thereby, in order to set bounds, we consider the smaller secondary abundance of SIMPs. Presumably most primordial SIMPs still are in galactic clouds, and the Earth is big enough to capture all SIMPs encountered along its trajectory. The total energy stored in captured SIMPs likely exceeds the energy of the world fossil fuel reserve by $10^{4}$. What happens after capture is unclear. If SIMPs do not bind in nuclei, they sink in the Earth's ocean and crust with drift velocity $v \sim 0.2 \mathrm{~km} / \mathrm{yr}$, such that their ground-level abundance is much below existing bounds. They can be searched for through dedicated enrichment processes and Rutherford backscattering experiments. If instead SIMPs bind within nuclei, electromagnetic interactions keep them in the crust since the crust becomes geologically stable. Then, the local SIMP density can be comparable to present bounds, depending on the capture cross section by nuclei, which is highly uncertain. 
SIMP searches have been also performed in meteorites, where SIMPs cannot sink. Despite this, meteorites are made of heavy elements synthesized by stars: primordial SIMPs sank to the center of stars, and never come back. The secondary abundance of SIMPs in meteorites depends on the SIMP capture cross section by individual nuclei, which is highly uncertain and possibly vanishing. Present bounds are satisfied assuming SIMP capture cross sections comparable to the one of neutrons with similar $\mathrm{MeV}$ energy, $\sigma_{\text {capture }} \sim 0.01 / \Lambda_{\mathrm{QCD}}^{2}$.

In conclusion, colored DM seems still allowed, although close to various bounds. Direct detection seems to provide the strongest and safest probe.

We discussed the apparently nicer model of colored DM: a neutral Dirac fermion $\mathcal{Q}$ in the adjoint representation of color. A scalar would give a similar phenomenology, and the DM abundance would be reproduced thermally for a similar $M_{\mathcal{Q}} \sim 12.5 \mathrm{TeV}$. A smaller mass would be obtained for quorns in the fundamental $\mathrm{SU}(3)_{\mathrm{c}}$, although the mass of the quorn-onlyum DM state $\mathcal{Q Q} \mathcal{Q}$ would be $M_{\mathrm{DM}} \approx 3 M_{\mathcal{Q}}$. In models where $\mathcal{Q}$ has an asymmetry, the DM abundance can be obtained for lower $M_{\mathcal{Q}}$.

Finally, we notice that the fall of free $\mathcal{Q}$ down to deep multi- $\mathcal{Q}$ bound states occurs around the QCD phase transition out of thermal equilibrium. It could thereby contribute to baryogengesis, provided that violation of baryon number can be added at an acceptable model, possibly assuming that $B$ is a gauge symmetry spontaneously broken giving rise to processes that violate $\Delta B \neq 1$.

\section{ACKNOWLEDGMENTS}

This work was supported by the ERC Grant No. NEONAT 669668. We thank Luca di Luzio, Gian Giudice, Paolo Panci, Maxim Pospelov and Antonio Vairo for very useful discussions. A.S. thanks CERN cafeteria for prompting this paper by selling a tasteless food named quorn.

\section{APPENDIX A: HYDROGEN DECAY RATES}

We summarize the known results for the hydrogen decay rates in dipole tree-level approximation [48]. We denote the initial state as $(n, \ell)$, and the final states as $\left(n^{\prime}, \ell^{\prime}\right)$. Their energy gap is

$$
\Delta E\left(n, n^{\prime}\right)=\frac{\alpha^{2} \mu}{2}\left(\frac{1}{n^{2}}-\frac{1}{n^{\prime 2}}\right),
$$

where $\mu$ is the reduced mass. The spontaneous emission rate, in dipole approximation, is

$\Gamma\left(n, \ell \rightarrow n^{\prime}, \ell^{\prime}\right)=\frac{4 \alpha}{3} \frac{\Delta E^{3}}{2 \ell+1} \sum_{m, m^{\prime}}\left|\left\langle n^{\prime}, \ell^{\prime}, m^{\prime}|\vec{r}| n, \ell, m\right\rangle\right|^{2}$.

Selection rules imply $\Delta \ell= \pm 1$, and the matrix elements are

$\sum_{m^{\prime}}\left|\left\langle n^{\prime}, \ell-1, m^{\prime}|\vec{r}| n, \ell, m\right\rangle\right|^{2}=\frac{\ell+1}{2 \ell+1} \frac{1}{(\alpha \mu)^{2}}\left(R_{n, \ell}^{n^{\prime}, \ell-1}\right)^{2}$,

$\sum_{m^{\prime}}\left|\left\langle n^{\prime}, \ell, m^{\prime}|\vec{r}| n, \ell-1, m\right\rangle\right|^{2}=\frac{\ell}{2 \ell+1} \frac{1}{(\alpha \mu)^{2}}\left(R_{n, \ell-1}^{n^{\prime}, \ell}\right)^{2}$,

where

$$
R_{n \ell}^{n^{\prime} \ell^{\prime}}=\int_{0}^{\infty} d r r^{3} R_{n \ell} R_{n^{\prime} \ell^{\prime}}
$$

with $R_{n \ell}(r)$ being the radial part of the hydrogen wave function. These integrals are given by

$$
\begin{aligned}
R_{n \ell}^{n^{\prime}, \ell-1}= & \frac{(-1)^{n^{\prime}-\ell}}{4(2 \ell-1) !} \sqrt{\frac{\left(n^{\prime}+\ell-1\right) !(n+\ell) !}{\left(n^{\prime}-\ell\right) !(n-\ell-1) !}} \frac{\left(4 n n^{\prime}\right)^{\ell+1}\left(n-n^{\prime}\right)^{n+n^{\prime}-2 \ell-2}}{\left(n+n^{\prime}\right)^{n+n^{\prime}}} \\
& \times\left[{ }_{2} F_{1}\left(-n+\ell+1,-n^{\prime}+\ell, 2 \ell,-\frac{4 n n^{\prime}}{\left(n-n^{\prime}\right)^{2}}\right)-\right. \\
& \left.+\left(\frac{n-n^{\prime}}{n+n^{\prime}}\right)_{2}^{2} F_{1}\left(-n+\ell-1,-n^{\prime}+\ell, 2 \ell,-\frac{4 n n^{\prime}}{\left(n-n^{\prime}\right)^{2}}\right)\right],
\end{aligned}
$$

where ${ }_{2} F_{1}$ is the Hypergeometric2F1 function. A similar formula can be obtained for $R_{n, \ell-1}^{n^{\prime}, \ell}$ by the interchange of the indices $n$ and $n^{\prime}$. The total decay rate and energy loss rate from an initial state $(n, \ell)$ is obtained by summing over all available lower-energy states with $n^{\prime}<n$.

\section{APPENDIX B: TOY REDECOUPLING}

We here show that the chromodark-synthesis mechanism is absolutely unavoidable by discussing a toy model that allows us to analytically understand some of its features. We consider formation of one bound state $B_{\mathcal{Q Q}}$ containing 
two DM quarks $\mathcal{Q}$ from two bound states $B_{\mathcal{Q}}$ containing one DM quark, ${ }^{10}$

$$
B_{\mathcal{Q}}+B_{\mathcal{Q}} \leftrightarrow B_{\mathcal{Q Q}}+X,
$$

where $X$ denotes any other SM particles, such as pions. We define $\delta \equiv 2 M_{B_{\mathcal{Q}}}-M_{B_{\mathcal{Q}}}$. In the real situation described in Sec. III, many bound states with a semiclassical discretuum of binding factors $\delta$ can be produced. We simplify the problem by considering just one of them, with $\delta \sim \Lambda_{\mathrm{QCD}}$ such that the QCD cross section for the above process is large, $\sigma_{\mathcal{Q Q}} \sim 1 / \delta^{2}$. One then reaches thermal equilibrium

$$
\frac{n_{B_{\mathcal{Q}}}}{n_{B_{\mathcal{Q}}}^{2}}=\frac{n_{B_{\mathcal{Q}}}^{\mathrm{eq}}}{n_{B_{\mathcal{Q}}}^{\mathrm{eq} 2}}=\frac{g_{B_{\mathcal{Q}}}}{g_{B_{\mathcal{Q}}}^{2}}\left(\frac{4 \pi}{M_{\mathcal{Q}} T}\right)^{3 / 2} e^{\delta / T} .
$$

This means that the $B_{\mathcal{Q}}$ dominantly form $B_{\mathcal{Q Q}}$ at the redecoupling temperature

$$
T_{\text {redec }}=\frac{\delta}{A} \quad \text { where } A=\ln \frac{Y_{\pi}}{Y_{\mathcal{Q}}} \sim 40
$$

is an entropy factor that describes how much formation of $B_{\mathcal{Q Q}}$ gets delayed by having a plasma with many more particles $X$ that can break it, than particles $B_{\mathcal{Q}}$ that can form it. This is analogous to how $e, p$ bind in hydrogen at $T \lesssim \delta / \ln \left(n_{\gamma} / n_{p}\right)$, and to how $p, n$ bind in deuterium at $T \lesssim \delta / \ln \left(n_{\gamma} / n_{p}\right)$, where $\delta$ are the binding energies of hydrogen and deuterium respectively. ${ }^{11}$

In the toy model, the residual density of $B_{\mathcal{Q}}$ is estimated as its thermal equilibrium value at the redecoupling temperature where the interaction rate $\Gamma_{\mathcal{Q Q}} \sim n_{B_{\mathcal{Q}}} \sigma_{\mathcal{Q Q}} v_{\text {rel }}$ for the process of Eq. (B1) becomes smaller than the Hubble rate. Imposing $\Gamma_{\mathcal{Q Q}} \sim H$ with $H \sim T^{2} / M_{\mathrm{Pl}}, v_{\text {rel }} \sim \sqrt{T / M_{\mathcal{Q}}}$ and $n_{B_{\mathcal{Q}}} \sim Y_{B_{\mathcal{Q}}} T^{3}$ gives

$$
\begin{aligned}
\left.Y_{B_{\mathcal{Q}}}^{\text {relic }}\right|_{\text {toy }} & \sim \frac{1}{\sigma_{\mathcal{Q} \mathcal{Q}} M_{\mathrm{Pl}} T_{\text {redec }} \sqrt{T_{\text {redec }} / M_{\mathcal{Q}}}} \\
& \sim A^{3 / 2} \frac{\sqrt{\delta M_{\mathcal{Q}}}}{M_{\mathrm{Pl}}} \sim 10^{-16} \sqrt{\frac{M_{\mathcal{Q}}}{10 \mathrm{TeV}} \frac{\delta}{\Lambda_{\mathrm{QCD}}}} .
\end{aligned}
$$

This shows that reannihilation is dominated by bound states with smaller $\delta \sim \Lambda_{\mathrm{QCD}}$, rather than by deep states. In the

\footnotetext{
${ }^{10}$ Similar considerations apply to formation of $B_{\mathcal{Q Q}}$ from free $\mathcal{Q}$ at $T \gtrsim \Lambda_{\mathrm{QCD}}$, but this phase is not relevant for the final DM abundance.

${ }^{11}$ In the numerical computation such an entropy factor was accounted in Sec. II E by imposing a small time allowed to radiate enough energy down to an unbreakable state. To keep the argument simple we here ignore the Boltzmann suppression in the $\pi$ abundance at $T \lesssim m_{\pi}$ (in the full numerical computation this is taken into account and increases the $\sigma_{\text {fall }}$ computed in Sec. II E, consequently suppressing the hybrid abundances).
}

full computation many bound states contribute to the depletion of $Y_{B_{Q}}$, which gets about 2 orders of magnitude smaller than the toy-model estimate of Eq. (B4). In turn, the unavoidable toy value is much smaller than what is obtained by including only perturbative QCD annihilations at $T \sim T_{\mathrm{dec}} \gg \Lambda_{\mathrm{QCD}}$.

\section{APPENDIX C: CHROMOPOLARIZABILITY OF $\mathcal{Q} \mathcal{Q}$ DM}

Equation (39) provides the formula for the polarizability of a QCD bound state. We here evaluate it for our DM, the $\mathcal{Q} \mathcal{Q}$ singlet bound state $|B\rangle=\left|1, s, \alpha_{\text {eff }}\right\rangle$ with energy $E_{10}=-\alpha_{\mathrm{eff}}^{2} M_{Q} / 4$, where $\alpha_{\mathrm{eff}}=3 \alpha_{3}$. By emitting a gluon it becomes a $p$-wave octet, with free Hamiltonian $H_{8}=$ $\vec{p}^{2} / M_{\mathcal{Q}}-\alpha_{8} / r$ where $\alpha_{8}=3 \alpha_{3} / 2$, whose eigenvalues are $E_{8 n}=-\alpha_{8}^{2} M_{Q} / 4 n^{2}$ for bound states and $\vec{p}^{2} / M_{\mathcal{Q}}$ for positive energy states. To evaluate the matrix element in Eq. (39) we insert the completeness relation for the octet eigenstates

$$
\begin{aligned}
\mathbf{1}_{8}= & \sum_{n, \ell, m}\left|n, \ell, m, \alpha_{8}\right\rangle\left\langle n, \ell, m, \alpha_{8}\right| \\
& +\frac{1}{3} \sum_{\ell, m} \int \frac{d^{3} p}{(2 \pi)^{3}}\left|\vec{p}, \ell, m, \alpha_{8}\right\rangle\left\langle\vec{p}, \ell, m, \alpha_{8}\right|
\end{aligned}
$$

where the first (second) term is the contribution from bound (free) states. The factor $1 / 3$ is introduced not to double count the angular momentum states. In coordinate space $\langle\vec{r} \mid n, \ell, m\rangle=R_{n \ell}(r) Y_{\ell m}(\theta, \phi)$ for bound states and $\langle\vec{r} \mid \vec{p}, \ell, m\rangle=R_{p \ell}(r) Y_{\ell m}(\theta, \phi)$ for continuum positive energy states, where $Y_{\ell m}(\theta, \phi)$ are spherical harmonics. The Coulombian wave functions are

$$
\begin{aligned}
R_{n \ell \alpha_{i}}(r)= & \left(\frac{2}{n a_{i}}\right)^{3 / 2} \sqrt{\frac{(n-\ell-1) !}{2 n(n+\ell) !}} \\
& \times e^{-r / n a_{i}}\left(\frac{2 r}{n a_{i}}\right)^{\ell} L_{n-\ell-1}^{2 \ell+1}\left(\frac{2 r}{n a_{i}}\right), \\
R_{p \ell \alpha_{i}}(r)= & \sqrt{4 \pi} \sqrt{2 \ell+1} \frac{\Gamma\left[1+\ell-i / a_{i} p\right]}{\Gamma[2(\ell+1)]} \\
& \times e^{\pi /\left(2 a_{i} p\right)} e^{-i p r}(2 i p r)^{\ell} \\
& \times{ }_{1} F_{1}\left[1+\ell+\frac{i}{a_{i} p}, 2(\ell+1), 2 i p r\right],
\end{aligned}
$$

where ${ }_{1} F_{1}$ is the HypergeometriclF1 function; $a_{i}=2 /\left(\alpha_{i} M_{\mathcal{Q}}\right)$ are the Bohr radii in each channel with effective coupling $\alpha_{i}=\left\{\alpha_{\text {eff }}, \alpha_{8}\right\}$ and $L_{n-\ell-1}^{2 \ell+1}$ are Laguerre polynomials.

Angular momentum conservation implies that only $p$-wave intermediate states contribute to the polarizability. The bound-state contribution thereby is 


$$
\left\langle B\left|\vec{r} \frac{1}{H_{8}-E_{10}} \vec{r}\right| B\right\rangle_{\text {bound }}=\sum_{n \geq 2} \frac{\left|\left\langle 1, s, \alpha_{\mathrm{eff}}|\vec{r}| n, p, \alpha_{8}\right\rangle\right|^{2}}{E_{8 n}-E_{10}},
$$

where the matrix element is

$$
\left|\left\langle 1, s, \alpha_{1}|\vec{r}| n, p, \alpha_{8}\right\rangle\right|^{2}=\left|\int_{0}^{\infty} d r r^{3} R_{10 \alpha_{\mathrm{eff}}}(r) R_{n 1 \alpha_{8}}(r)\right|^{2} .
$$

Performing numerically the integral and the sum one finds

$$
\left\langle B\left|\vec{r} \frac{\alpha_{3}}{H_{8}-E_{10}} \vec{r}\right| B\right\rangle_{\text {bound }}=0.359 a^{3} .
$$

The contribution of unbound $E>0$ intermediate states is found generalizing the formulas in [49],

$$
\begin{aligned}
\left\langle B\left|\vec{r} \frac{\alpha_{3}}{H_{8}-E_{10}} \vec{r}\right| B\right\rangle_{\text {free }} \\
=\frac{1}{3} \int \frac{d^{3} p}{(2 \pi)^{3}} \frac{\alpha_{3}}{p^{2} / M_{\mathcal{Q}}-E_{10}}\left|\int_{0}^{\infty} d r r^{3} R_{10 \alpha_{\mathrm{eff}}}(r) R_{p 1 \alpha_{8}}(r)\right|^{2} \\
=a^{3} \frac{512}{C} \rho(\rho+2)^{2} \\
\quad \times \int_{0}^{\infty} p^{3} \frac{\left(1+\rho^{2} / p^{2}\right) e^{-4 \rho / p \arctan p}}{\left(e^{2 \pi \rho / p}-1\right)\left(1+p^{2}\right)^{7}} d p=1.17 a^{3},
\end{aligned}
$$

where $C=3$ and $\rho=-\alpha_{8} / \alpha_{\text {eff }}=-1 / 2$ for our color octets. In the case of the hydrogen atom $(C=1, \rho=-1)$ one finds [39] $\left.c_{E}\right|_{\text {hydrogen }}=8 \pi(5.49+1.26) a^{3} / 3=18 \pi a^{3}$. The $\mathcal{Q Q}$ chromopolarizability is smaller than what is suggested by a naive rescaling of the Abelian result computed for the hydrogen atom $\left.c_{E}\right|_{\text {naive }}=18 \pi a^{3} C /\left(N_{c}^{2}-1\right)=6.75 \pi a^{3}$.
[1] E. Witten, Cosmic separation of phases, Phys. Rev. D 30, 272 (1984).

[2] G. R. Farrar, Stable Sexaquark, arXiv:1708.08951.

[3] M. Cirelli, N. Fornengo, and A. Strumia, Minimal dark matter, Nucl. Phys. B753, 178 (2006).

[4] M. L. Perl, E. R. Lee, and D. Loomba, Searches for fractionally charged particles, Annu. Rev. Nucl. Part. Sci. 59, 47 (2009).

[5] P. J. Fox, A. E. Nelson, and N. Weiner, Dirac gaugino masses and supersoft supersymmetry breaking, J. High Energy Phys. 08 (2002) 035.

[6] J. E. Kim, Weak Interaction Singlet and Strong $C P$ Invariance, Phys. Rev. Lett. 43, 103 (1979); M. A. Shif-man, A. I. Vainshtein, and V.I. Zakharov, Can confinement ensure natural $C P$ invariance of strong interactions? Nucl. Phys. B166, 493 (1980).

[7] M. Farina, D. Pappadopulo, and A. Strumia, A modified naturalness principle and its ex-perimental tests, J. High Energy Phys. 08 (2013) 022.

[8] E. Eichten, K. Gottfried, T. Kinoshita, J. B. Kogut, K. D. Lane, and T.-M. Yan, The Spectrum of Charmonium, Phys. Rev. Lett. 34, 369 (1975).

[9] P. Bicudo, The QCD string tension curve, the ferromagnetic magnetization, and the quark-antiquark confining potential at finite temperature, Phys. Rev. D 82, 034507 (2010); P. Petreczky, Lattice QCD at nonzero temperature, J. Phys. G 39, 093002 (2012); S. Aoki et al., Review of lattice results concerning low-energy particle physics, Eur. Phys. J. C 77, 112 (2017).

[10] H. S. Bali, Casimir scaling of SU(3) static potentials, Phys. Rev. D 62, 114503 (2000).

[11] G. S. Bali, Casimir scaling or flux counting?, Nucl. Phys. B, Proc. Suppl. 83-84, 422 (2000).
[12] R. L. Hall, Simple eigenvalue formula for the Coulomb plus linear potential, Phys. Rev. D 30, 433 (1984).

[13] J. Kang, M. A. Luty, and S. Nasri, The relic abundance of long-lived heavy colored particles, J. High Energy Phys. 09 (2006) 086.

[14] M. Kusakabe and T. Takesako, Resonant annihilation of long-lived massive colored particles through hadronic collisions, Phys. Rev. D 85, 015005 (2012).

[15] G. Bhanot and M.E. Peskin, Short-distance analysis for heavy quark systems. 2. Applications, Nucl. Phys. B156, 391 (1979).

[16] A. Mitridate, M. Redi, J. Smirnov, and A. Strumia, Cosmological implications of dark matter bound states, J. Cosmol. Astropart. Phys. 05 (2017) 006; a sign was corrected in agreement with A. V. Manohar and I. W. Stewart, Running of the heavy quark production current and $1 /|\mathrm{k}|$ potential in QCD, Phys. Rev. D 63, 054004 (2001).

[17] A. Arvanitaki, C. Davis, P. W. Graham, A. Pierce, and J. G. Wacker, Limits on split supersymmetry from gluino cosmology, Phys. Rev. D 72, 075011 (2005).

[18] C. B. Dover, T. K. Gaisser, and G. Steigman, Cosmological Constraints on New Stable Hadrons, Phys. Rev. Lett. 42, 1117 (1979).

[19] D. A. Dicus and V.L. Teplitz, Primordial Synthesis of Anomalous Nuclei, Phys. Rev. Lett. 44, 218 (1980).

[20] R. N. Mohapatra and V. L. Teplitz, Primordial Nucleosynthesis Constraint on Massive, Stable, Strongly Interacting Particles, Phys. Rev. Lett. 81, 3079 (1998).

[21] M. Kusakabe, T. Kajino, T. Yoshida, and G. J. Mathews, Effect of long-lived strongly interacting relic particles on big bang nucleosynthesis, Phys. Rev. D 80, 103501 (2009). 
[22] E. Epelbaum, H.-W. Hammer, and U.-G. Meissner, Modern theory of nuclear forces, Rev. Mod. Phys. 81, 1773 (2009).

[23] E. Epelbaum, Nuclear forces from chiral effective field theory: A primer, arXiv:1001.3229.

[24] R. Machleidt, The high precision, charge dependent Bonn nucleon-nucleon potential (CD-Bonn), Phys. Rev. C 63, 024001 (2001).

[25] T. Miyamoto et al., $\Lambda_{c} N$ interaction from lattice QCD and its application to $\Lambda_{c}$ hypernuclei, Nucl. Phys. A971, 113 (2018).

[26] G. R. Farrar and G. Zaharijas, Nonbinding of avor-singlet hadrons to nuclei, Phys. Lett. B 559, 223 (2003).

[27] R. H. Cyburt, B. D. Fields, V. Pavlidou, and B. D. Wandelt, Constraining strong baryon dark matter interactions with primordial nucleosynthesis and cosmic rays, Phys. Rev. D 65, 123503 (2002).

[28] G. D. Starkman, A. Gould, R. Esmailzadeh, and S. Dimopoulos, Opening the window on strongly interacting dark matter, Phys. Rev. D 41, 3594 (1990).

[29] M. W. Goodman and E. Witten, Detectability of certain dark matter candidates, Phys. Rev. D 31, 3059 (1985).

[30] G. D. Mack, J. F. Beacom, and G. Bertone, Towards closing the window on strongly interacting dark matter: Farreaching constraints from Earth's heat flow, Phys. Rev. D 76, 043523 (2007).

[31] S. Burdin, M. Fairbairn, P. Mermod, D. Milstead, J. Pinfold, T. Sloan, and W. Taylor, Noncollider searches for stable massive particles, Phys. Rep. 582, 1 (2015).

[32] S. Polikanov, C. S. Sastri, G. Herrmann, K. Lutzenkirchen, M. Overbeck, N. Trautmann, A. Breskin, R. Chechik, and Z. Fraenkel, Search for supermassive nuclei in nature, Zeitschrift fuer Physik A Hadrons and Nuclei 338, 357 (1991).

[33] P. F. Smith, J. R. J. Bennett, G. J. Homer, J. D. Lewin, H. E. Walford, and W. A. Smith, A search for anomalous hydrogen in enriched D2O, using a time-of-flight spectrometer, Nucl. Phys. B206, 333 (1982).

[34] M. Heine et al., Determination of the neutron-capture rate of 17C for r-process nucleosynthesis, Phys. Rev. C 95, 014613 (2017).

[35] T. K. Hemmick et al., A search for anomalously heavy isotopes of low Z nuclei, Phys. Rev. D 41, 2074 (1990).

[36] E. B. Norman, S. B. Gazes, and D. A. Bennett, Searches for Supermassive X Particles in Iron, Phys. Rev. Lett. 58, 1403 (1987).

[37] J. Hisano, R. Nagai, and N. Nagata, Effective theories for dark matter nucleon scattering, J. High Energy Phys. 05 (2015) 037.

[38] Xenon1T Collaboration, First Dark Matter Search Results from the Xenon1T Experiment, Phys. Rev. Lett. 119, 181301 (2017).
[39] M.E. Peskin, Short-distance analysis for heavy quark systems. 1. Diagrammatics, Nucl. Phys. B156, 365 (1979); M. B. Voloshin, On dynamics of heavy quarks in nonperturbative QCD vacuum, Nucl. Phys. B154, 365 (1979); A. Dalgarno and J. T. Lewis, The exact calculation of long-range forces between atoms by perturbation theory, Proc. R. Soc. 233, 70 (1955).

[40] CTA Collaboration, Prospects for indirect dark matter searches with the Cherenkov Telescope Array (CTA), Proc. Sci., ICRC2015 (2015) 1203 [arXiv:1508.06128].

[41] M. Cirelli, G. Corcella, A. Hektor, G. Hütsi, M. Kadastik, P. Panci, M. Raidal, F. Sala, and A. Strumia, PPPC 4 DM ID: A poor particle physicist cookbook for dark matter indirect detection, J. Cosmol. Astropart. Phys. 03 (2010) 051; P. Baratella, M. Cirelli, A. Hektor, J. Pata, M. Piibeleht, and A. Strumia, PPPC 4 DMv: A poor particle physicist cookbook for neutrinos from dark matter annihilations in the Sun, J. Cosmol. Astropart. Phys. 03 (2014) 053.

[42] L. Schiff, Quantum Mechanics (McGraw-Hill, 1968).

[43] H. Fujii and D. Kharzeev, Long-range forces of QCD, Phys. Rev. D 60, 114039 (1999).

[44] D. L. Morgan and V. W. Hughes, Atomic processes involved in matter-antimatter annihilation, Phys. Rev. D 2, 1389 (1970); Atom-antiatom interactions, Phys. Rev. A 7, 1811 (1973); P. Froelich, S. Jonsell, A. Saenz, B. Zygelman, and A. Dalgarno, Hydrogen-Antihydrogen Collisions, Phys. Rev. Lett. 84, 4577 (2000); S. Jonsell, A. Saenz, and P. Froelich, Low-energy hydrogen antihydrogen collisions, Nucl. Phys. A663, 959c (2000).

[45] See e.g., CMS Collaboration, Search for heavy stable charged particles with $12: 9 \mathrm{fb}^{-1}$ of 2016 data, Report No. CMS-PAS-EXO-16-036; ATLAS Collaboration, Reinterpretation of searches for supersymmetry in models with variable R-parity-violating coupling strength and longlived R-hadrons, Report No. ATLAS-CONF-2018-003.

[46] G. Grilli di Cortona, E. Hardy, and A. J. Powell, Dirac vs Majorana gauginos at a $100 \mathrm{TeV}$ collider, J. High Energy Phys. 08 (2016) 014.

[47] J. Ellis, F. Luo, and K. A. Olive, Gluino coannihilation revisited, J. High Energy Phys. 09 (2015) 127.

[48] For recent works see S. Olszewski and T. Kwiatkowski, Semiclassical approach to intensity spectrum of atomic hydrogen, Z. Phys. D 21, 201 (1991); M. Seidl and P. O. Lipas, Semiclassical interpretation of spontaneous transitions in the hydrogen atom, Eur. J. Phys. 17, 25 (1996). See also the references therein.

[49] N. Brambilla, G. Krein, J. Tarrús Castellá, and A. Vairo, Long-range properties of $1 \mathrm{~S}$ bottomonium states, Phys. Rev. D 93, 054002 (2016). 